\title{
THE WORLDSHEET PERSPECTIVE OF T-DUALITY SYMMETRY IN STRING THEORY
}

\author{
Jnanadeva Maharana ${ }^{1}$ \\ E-mail maharana@iopb.res.in \\ October 1, 2018 \\ Institute of Physics \\ Bhubaneswar - 751005 \\ India \\ and \\ NISER, Bhubaneswar 751005, India
}

\begin{abstract}
The purpose of this article is to present a pedagogical review of T-duality in string theory. The evolution of the closed string is envisaged on the worldsheet in the presence of its massless excitations. The duality symmetry is studied when some of the spacial coordinates are compactified on d-dimensional torus, $T^{d}$. The known results are reviewed to elucidate that equations of motion for the compact coordinates are $O(d, d)$ covariant, $d$ being the number of compact directions. Next, the vertex operators of excited massive levels are considered in a simple compactification scheme. It is shown that the vertex operators for each massive level can be cast in a T-duality invariant form in such a case. Subsequently, the duality properties of superstring is investigated in the NSR formulation for the massless backgrounds such as graviton and antisymmetric tensor. The worldsheet superfield formulation is found to be very suitable for our purpose. The Hassan-Sen compactification is adopted and it is shown that the worldsheet equations of motion for compact superfields are $O(d, d)$ covariant when the backgrounds are independent of superfields along compact directions. The vertex operators for excited levels are presented in the NS-NS sector and it is shown that they can be cast in T-duality invariant form for the case of Hassan-Sen compactification scheme. An illustrative example is presented to realize our proposal.
\end{abstract}

\footnotetext{
${ }^{1}$ In Memory of Sumitra Maharana

Invited Review Article for International Journal of Modern Physics A
} 


\section{Introduction}

Our principal endeavor in physics is to comprehend microscopic laws of Nature from a few fundamental principles. Therefore the goal to unify the four basic forces is of paramount importance. The standard model of particle physics is described by the Electroweak theory which is unification of electromagnetic and weak forces and the Quantum Chromodynamics (QCD) is the underlying theory of the interaction of quarks and gluons, which is responsible for strong nuclear force. The standard model has been tested to great degree of accuracy. There are evidences which point towards unification of three of the four fundamental interactions i.e. weak, electromagnetic and strong forces. Moreover, there are compelling arguments for supersymmetry which might be discovered in accessible high energy collider experiments. However, experimental discovery of supersymmetric partner particles is still awaited. It has been a cherished dream of generations of physicists to unify the forces of Nature. It is recognized that, within the perturbation theoretic frame work, the unification of the four forces encounters some difficulties in the field theoretic approach. String theory is a radical step for accomplishing this goal. It incorporates gravitational interaction and it addresses many important issues pertaining to quantum gravity. The computation of Bekenstein-Hawking entropy associated with a special class of stringy black holes as derived from a microscopic theory, like string theory, is one of the major achievements in this frame work. Furthermore, the decay rates of special class of stringy black holes to stringy BPS black holes have been successfully evaluated and there is no conflict with unitarity of S-matrix in this context which resolves one of important issues in black hole physics. It is also expected that string theory will provide insights into the conceptual frame works related to the creation of the Universe and its evolution in early epochs. String theory has provided a basis to explain the mechanism of cosmological inflation. Although the standard model of particle physics is so well tested, there are several reasons to believe that standard model is incomplete to some extent. For example the Yukawa couplings of the fundamental fermions in the standard model, which generate their masses, are arbitrary parameters. There are several other issues which has stimulated research to expound ideas which are beyond the paradigms of the standard model. It is hoped that string theory, being a unified theory of fundamental forces, will address all these questions collectively. Substantial progress has been made in order to bring string theory closer to low energy theories of elementary particles. Thus phenomenological aspects of string theory has attracted considerable attention. Although the standard model of particle physics, described by $S U(3)_{c} \otimes S U(2) \otimes U(1)$, is yet to emerge from string theory, interesting developments are going on to achieve this goal. At this juncture, string theory is in an interesting phase. There are important developments in the formal aspects which are bordering and influencing research in certain areas of mathematics. On the other hand there are efforts to establish connections with the phenomenology of low energy particle physics. 
The past history as a guide has taught us that symmetry is a guiding principle to understand physical phenomena and has played crucial role in unraveling the laws of Nature. For example, the gauge symmetries have been utilized as cardinal principle to construct the standard model. It is well known that perturbatively consistent string theories live in critical dimensions: bosonic strings in 26 dimensions and superstrings in 10 dimensions. Moreover, in the critical dimensions, there are five perturbatively distinct superstring theories. As alluded to above, one of the major efforts is to construct theories in four spacetime dimensions in order to establish connections with the standard model. Therefore, it is proposed that the extra dimensions (six of them) are very small. In other words the ten dimensional theory is compactified to four dimensions where the extra spatial six dimensions are so small that we are unable to probe them using present accessible energy scales. The idea of compactification goes back to Kaluza and Klein. In the string theoretic scenario, it has very important and interesting implications. Since string is a one dimensional object it can wrap around the 'internal' compactified directions. We do not envisage this possibility in field theoretic description of a point particle. Consequently, a rich symmetry structure emerges which is a special characteristic of string theory. Although there are five different superstring theories in critical dimensions when these theories are compactified to lower spacetime dimensions through compactification of extra spatial dimensions, we discover presence of novel symmetries. Some of these symmetries have field theoretic analog. We also encounter new types of symmetries which are special attributes of string theories. The dualities symmetries, among these, are very important. The duality symmetries relate the five string theories when we compactify them to lower dimensions. Although the five superstring theories are perturbatively distinct in the critical dimension, $D=10$, in the lower spacetime dimensions they get related through the web of dualities. Therefore, it is believed that the five string theories might be different 'phases' of a unique fundamental theory, the M-theory. There are evidences that such theory might exist, however, a satisfactory construction of such a theory is lacking.

The goal of this article is modest and twofold and it is presented in a pedagogical manner. The introductory part contains elementary introduction to T-duality with some illustrative examples. It is presumed that the reader is familiar with the first quantized approach to string theory. It is expected that the reader has background of the mode expansions, computation of the energy levels in string theory and is familiar with results of two dimensional conformal field theory. Moreover, it is desirable that reader is acquainted with S-duality and T-duality. We shall focus our attention on the latter from the worldsheet perspective. An interested reader can use introductory sections of this article to study technical details in text books and review papers. The second part of this article is a personal point of view of the author. It has been argued that not only we encounter T-duality in the massless sector of the closed string but also massive excited levels of closed string are endowed with the T-duality symmetry. This conjecture is pursued in the worldsheet approach. More precisely, it is proposed 
that the vertex operators associated with the excited massive levels exhibit T-duality for a string compactified on a torus which will be stated in more detail in sequel. There are evidences in favor of this conjecture when vertex operators are studied in a simple compactification scheme. Subsequently, the technique is applied to NSR formalism of superstrings in the presence of NS-NS backgrounds. Thus the next two sections (III and IV) contain summary of my own results. However, I have presented the essential steps for the convenience of the reader.

In recent years a lot of attention has been focused on duality symmetries. There are excellent text books $[1,2,3,4,5,6,7]$ and review articles $[8,9,10,11,12,13,14,15,16]$ on this topic. For an article of this scope and size it is not possible to provide complete references to all publications. Therefore, keeping in mind the size of the article, I have provided references to books and review article and some of the articles published during the nascent period of the field. Any omission is neither intentional nor it is to deprive any author of due credit.

I would like to present my motivations for study of T-duality in a historical perspective. We recall that the idea of string theory was conceived from study of collisions of hadrons - strongly interacting particles in the S-matrix approach. In the low energy regime, the scattering amplitudes are dominated by nearby resonances and at high energy for small scattering angles they were described by power dependence in energy - the Regge behavior. The finite energy sum rules (FESR) connected these two seemingly different attributes of scattering amplitudes [17, 18, 19]. Therefore, the challenge was to construct an amplitude endowed with both these features, the so called duality property of that era. The model proposed by Veneziano [20] incorporated those attributes. Indeed construction of 4-point amplitude with the desired features led to the birth of string theory. Virasoro [21] proposed an amplitude with analogous features. Subsequently, multiparticle (n-particle onshell) scattering amplitudes were constructed. It was soon recognized that Veneziano-type amplitudes owe their origin to tree level open string amplitudes and those of Virasoro are derivable from the closed string theory. As I shall argue in sequel, this duality symmetry is encoded in the string worldsheet Hamiltonian in its simplest form. Furthermore, when we envisage evolution of a closed bosonic string in the presence of its massless excitation in some special circumstances the afore mentioned symmetry appears in a different garb. Therefore, my conjecture is that, in a very simple scenario, the duality symmetry should be exhibited in string theory when we consider the scattering of massive states. I provide evidences in favor of my conjecture in a restricted picture. The plan of the article is as fellows. In the next section we recall the rudimentary results of string theory which we need for our discussions. We introduce simple result of string theory as an illustrative example. Section II deals with compactification of a string on a torus. We show how T-duality acts in this theory. Furthermore, it is demonstrated that perturbative spectrum of the theory remains invariant under the T-duality. Next it is shown, in the Lagrangian formulation, how the string equations of motion can be cast in a duality covariant manner [22]. A brief excursion is made to 
study T-duality from the effective action point of view for the sake of completeness. The third section begins by motivation the reader about importance of massive excited states of strings although most of the works in string theory are focused on the massless sector since massive states are of the order of (generally) Planck mass and therefore, they are not so important in the low energy regime. Some of the well known results about vertex operators of massive states are recalled and the consequences of conformal invariance, in this context, are presented. A simple scheme is introduced to examine T-duality properties of first excited massive level [23], keeping in mind the remarks of the preceding paragraph. Furthermore, an analogy is drawn with vertex operator of massless level when some of the spatial dimensions of the string are compactified. It is argued that such a simple proposal is inadequate to study T-duality for vertex operators of arbitrary massive levels since the vertex operators assume rather complicated form and have large number of terms. However, encouraged by initial results for first excited massive state, a prescription is introduced in order to construct manifestly duality invariant vertex operators for a string whose d-coordinates are compactified on a torus $T^{d}$. The fourth Section is devoted to study of T-duality symmetry for NSR string in the NS-NS backgrounds when the theory is compactified on $T^{d}$. We present two interesting results. We resort to the woldsheet superfield formulation to study T-duality. In the first step, we consider, NSR string in its massless backgrounds in the NS-NS sector, i.e. graviton and antisymmetric tensor fields which are independent of superfields along compact directions. Until recently, it was not shown explicitly that worldsheet equations of motion in this case, i.e. for compact coordinates, can be expressed in T-duality covariant form. However, for closed bosonic string the equations of motion can be expressed in duality covariant form [22] as we shall see later. We achieved this objective by studying the equations of motion in the superfield formalism. With this formulation in place, the path is paved to construct vertex operators for massive excited states of the NSR string in the NS-NS sector. We suitably modify the corresponding formulation for the closed bosonic string to construct T-duality invariant vertex operators for NSR string in its NS-NS sector. We also present an explicit example of our result for type IIB theory, in its NS-NS sector when the theory is compactified on $A d S_{3} \otimes S^{3} \otimes T^{4}$. The last section summarizes our results and future directions. In this section an attempt is made to establish connection between worldsheet T-duality and recently formulated double field theory.

\section{T-duality Compactified String: Massless Sector}

The rich symmetry contents of string theory have played a cardinal role in understanding diverse attributes of string theory and have provided deep incisive insights into its dynamics. The target space duality (T-duality) is a very special feature of string theory and has attracted considerable attentions over two decades 
$[1,2,3,4,5,6,7,8,9,10,11,12,14,15,16]$. This symmetry owes its existence to one-dimensional nature of string and we do not encounter the analog of T-duality in field theoretic description of a point particle. Therefore, it is natural to explore the symmetry from the perspective of the worldsheet. The T-duality symmetry, associated with closed bosonic string, has been investigated from the worldsheet perspective in a general framework almost two decades ago [22]. The closed bosonic string was considered in the presence of its massless backgrounds when $d$ of its target space coordinates were compactified on a torus. The backgrounds along compact directions were allowed to depend on noncompact string coordinates and were assumed to be independent of compact coordinates. It was recognized that the worldsheet equations of motion are conserved currents along compact directions. The target space duality (T-duality) is a very special feature of string theory and has attracted considerable attentions over two decades $[9,10,11,12,14,8,15,16]$. We introduce dual coordinates corresponding to each compact coordinate and dual backgrounds to derive another set of equations of motion. The two sets of equations of motion are suitably combined to derived $O(d, d)$ covariant equations of motion [22] where $d$ is the number of compact coordinates.

On the other hand when T-duality is analyzed in a general setting its salient features and powerful applications are exhibited from the view point of the target space in the effective action approach. To recapitulate, when we envisage evolution of a closed string in the background of its massless excitations and demand (quantum) conformal invariance the backgrounds are constrained through the $\beta$-function equations which are computed perturbatively in the worldsheet $\sigma$-model approach. These equations of motion enable us to introduce the effective action whose variation reproduces the "equations of motion". Let us toroidally compactify the effective action to lower dimensions and examine symmetries of the reduced effective action. The reduced action can be cast in a manifestly $O(d, d)$ invariant form following the Scherk-Schwarz [24] dimensional reduction scheme if the theory is compactified on a d-dimensional torus $\left(T^{d}\right)$ and the massless backgrounds are independent of the compact coordinates whereas they carry spacetime dependence. The availability of a manifestly $O(d, d)$ invariant reduced effective action has been very useful to explore various aspects of string theory in diverse directions. The target space duality has attracted a lot of attentions from different perspectives over a long period. We refer to some early papers $[25,26,27,28,29,30,31,32,33,34,35,36,37,38,39,40,41,42]$ and interested reader may consult reviews for comprehensive list of papers $[9,10,11,12,14,8,15,16]$.

The strong-weak duality is a symmetry which relates weak coupling phase to the strong coupling phase [43, 44, 45, 46, 47]. This S-duality might relate weak coupling phase of a theory to the strong coupling phase of another theory. For example, heterotic string compactified on $T^{4}$ is S-dual to type IIA theory compactified on $K_{3}$. In certain cases they theory might be selfdual. A familiar example is $\mathcal{N}=4$ supersymmetric Yang-Mills theory. It is obvious that S-duality symmetry cannot be tested in the perturbative frame work. S-duality together with T-duality have proved to 
be a powerful tool to study string dynamics in diverse dimensions. We mention en passant that analog of S-duality is of importance in statistical mechanics where the temperature plays the role of coupling constant.

\subsection{T-Duality: The Woldsheet Description}

In this section we briefly recall some of the known and essential results of string theory. Our attention is focused on closed string theory. Let us consider the action of a closed string

$$
S=\frac{1}{2} \int d^{2} \sigma \gamma^{a b} \partial_{a} X^{\hat{\mu}} \partial_{b} X_{\hat{\mu}}
$$

where $\hat{\mu}, \hat{\nu}=0,1, \ldots . \hat{D}-1, \hat{D}$ being the number of spacetime dimensions. The above action is the so called gauge fixed action in the sense that the two dimensional metric is chosen to be the flat metric since we can transform any metric to a conformally flat metric and (classically) the conformal factor does not appear. Here $\gamma^{a b}$ is the two dimensional Lorentzian signature flat metric. We choose this metric convention here and everywhere. The equations of motion is

$$
\left(\frac{\partial^{2}}{\partial \tau^{2}}-\frac{\partial}{\partial \sigma^{2}}\right) X^{\hat{\mu}}=0
$$

For a free closed string, $X^{\hat{\mu}}$ is periodic in $\sigma$ and the solution can be decomposed into left and right movers i.e $X^{\hat{\mu}}=X_{L}^{\hat{\mu}}(\tau+\sigma)+X_{R}^{\hat{\mu}}(\tau-\sigma)$. The left and right movers are expanded in terms of the oscillators in addition to the zero modes in each sector. The reparametrization invariance symmetry on the worldsheet, in the quantum theory, requires that the string lives in twenty six spacetime dimensions.

The canonical Hamiltonian density is

$$
H_{c}=\frac{1}{2}\left(P^{2}+X^{\prime 2}\right)
$$

$P_{\hat{\mu}}$ being the canonical momentum and $X^{\prime \hat{\mu}}$ is derivative of string coordinate with respect to $\sigma$. Since $P_{\hat{\mu}}=\dot{X}_{\hat{\mu}}$, note that the Hamiltonian density remains invariant under $P \leftrightarrow X^{\prime}$, i.e. when $\tau \leftrightarrow \sigma$ are interchanged. This is the simplest example of T-duality for a closed string in flat spacetime geometry.

In the first quantized approach to string theory, the evolution of the string is envisaged in its massless excitations such as graviton, $G$ and antisymmetric tensor field, B, for a closed string. The worldsheet action assumes the form,

$$
L=\frac{1}{2} \int d^{2} \sigma\left(\gamma^{a b} G(X)_{\hat{\mu} \hat{\nu}}(X) \partial_{a} X^{\hat{\mu}} \partial_{b} X^{\hat{\nu}}+\epsilon^{a b} B(X) \hat{\mu} \hat{\nu} \partial_{a} X^{\hat{\mu}} \partial_{b} X^{\hat{\nu}}\right)
$$

The above action is a two dimensional $\sigma$-model action. The requirements of conformal invariance imposes constraints on the backgrounds $G$ and $B$ since the associated $\beta$ function must vanish. These constraints assume forms of differential equations for $G$ 
and $B$, the so called equations of motion.

In order to explore the world sheet duality symmetry in the simplest scenario, let us assume the backgrounds $G$ and $B$ to be constant i.e. independent of string coordinates $X^{\hat{\mu}}$. In this case the Hamiltonian density is expressed as

$$
H_{c}=\frac{1}{2} Z^{T} M(G, B) Z
$$

where

$$
Z=\left(\begin{array}{l}
P \\
X^{\prime}
\end{array}\right)
$$

and we have suppressed the indices.

$$
M=\left(\begin{array}{cc}
G^{-1} & -G^{-1} B \\
B G^{-1} & G-B G^{-1} B
\end{array}\right)
$$

is a symmetric $2 \hat{D} \times 2 \hat{D}$ matrix. $G$ and $B$ are constant backgrounds.

Note that under interchange $P \leftrightarrow X^{\prime}$, the Hamiltonian density remains invariant if we simultaneously transform $M \leftrightarrow M^{-1}$. The Hamiltonian density is also invariant under the following global $\mathbf{O}(\hat{\mathbf{D}}, \hat{\mathbf{D}})$ transformations: The $Z$-vector and $M$-matrix transform as

$$
Z \rightarrow \Omega_{0} Z, \quad M \rightarrow \Omega_{0} M \Omega_{0}^{T}, \eta_{0} \rightarrow \eta_{0}, \quad \Omega_{0} \in O(\hat{D}, \hat{D})
$$

where $\eta_{0}$ is the $O(\hat{D}, \hat{D})$ metric.

$$
\eta_{0}=\left(\begin{array}{ll}
0 & 1 \\
1 & 0
\end{array}\right)
$$

where $\mathbf{1}$ is $\hat{D} \times \hat{D}$ unit matrix. $\mathbf{Z}$ is $2 \hat{D}$-dimensional $O(\hat{D}, \hat{D})$ vector.

In presence of constant backgrounds, the woldsheet equations of motion for string coordinates $\left\{X^{\hat{\mu}}(\sigma, \tau)\right\}$ are set of conservation laws [56]

$$
\partial_{a} \mathcal{J}_{\hat{\mu}}^{a}=0
$$

as follows from (4) where the current is given by

$$
\mathcal{J}_{\hat{\mu}}^{a}=\gamma^{a b} G_{\hat{\mu} \hat{\nu}} \partial_{b} X^{\hat{\nu}}+\epsilon^{a b} B_{\hat{\mu} \hat{\nu}} \partial_{b} X^{\hat{\nu}}
$$

Thus locally, one can express the two dimensional current as:

$$
\gamma^{a b} \partial_{b} X^{\hat{\nu}} G_{\hat{\mu} \hat{\nu}}+\epsilon^{a b} \partial_{b} X^{\hat{\nu}} B_{\hat{\mu} \hat{\nu}}=\epsilon_{a b} \partial_{b} \tilde{X}_{\hat{\nu}}
$$

where $\left\{\tilde{X}_{\hat{\mu}}\right\}$ are set of dual coordinates. The next step is to introduce a set of auxiliary fields $U_{a}^{\hat{\mu}}$ and then define a dual action

$$
\tilde{S}=\int d^{2} \sigma\left(\frac{1}{2}\left[\left(\gamma^{a b} U_{a}^{\hat{\mu}} U_{b}^{\hat{\nu}} G_{\hat{\mu} \hat{\nu}}+\epsilon^{a b} U_{a}^{\hat{\mu}} U_{b}^{\hat{\nu}} B_{\hat{\mu} \hat{\nu}}\right]\right)\right.
$$


The associated equation of motion is

$$
\gamma^{a b} U_{b}^{\hat{\nu}} G_{\hat{\mu} \hat{\nu}}+\epsilon^{a b} U_{b}^{\hat{\nu}} B_{\hat{\mu} \hat{\nu}}-\epsilon^{a b} \partial_{b} \tilde{X}_{\hat{\mu}}=0
$$

If we identify $U_{a}^{\hat{\mu}}$ with $\partial_{a} X^{\hat{\mu}}$ then we recover original equations of motion. Thus we can use (14) to solve for $U_{a}^{\hat{\mu}}$ in terms of $\partial_{a} \tilde{X}^{\hat{\mu}}$

$$
U_{a}^{\hat{\mu}}=\left(\epsilon_{a}^{b} \mathcal{G}^{\hat{\mu} \hat{\nu}}+\delta_{a}^{b} \mathcal{B}^{\hat{\mu} \hat{\nu}}\right) \partial_{b} \tilde{X}_{\hat{\nu}}
$$

here we have introduced

$$
\mathcal{G}=\left(G-B G^{-1} B\right)^{-1}
$$

and

$$
\mathcal{B}=-G^{-1} B\left(G-B G^{-1} B\right)^{-1}
$$

Note that $\mathcal{G}$ and $\mathcal{B}$ are symmetric and antisymmetric tensors satisfying $(G+B)(\mathcal{G}+$ $\mathcal{B})^{-1}=1$. Once we substitute expression for $U_{a}^{\hat{\mu}}$ in $(13)$, the dual action assumes the following form

$$
\tilde{S}=\int d^{2} \sigma\left(\frac{1}{2}\left[\gamma^{a b} \partial_{a} \tilde{X}_{\hat{\mu}} \partial_{b} \tilde{X}_{\hat{\nu}} \mathcal{G}^{\hat{\mu} \hat{\nu}}+\epsilon^{a b} \partial_{a} \tilde{X}_{\hat{\mu}} \partial_{b} \tilde{X}_{\hat{\nu}} \mathcal{B}_{\hat{\mu} \hat{\nu}}\right]\right)
$$

We mention in passing that $\mathcal{G}$ and $\mathcal{B}$ are also constant dual backgrounds. Moreover, the $\tilde{X}$ equations of motion derived from $\tilde{S}$ are also conservation laws. Thus the two actions $S$ and $\tilde{S}$ give a pair of equations of motion. The two sets are equivalent in the sense that they describe the evolution of the same string theory and the actions are dual to each other. Since equations from (18) are worldsheet current conservation law we can identify (locally) the current as

$$
\epsilon^{a b} \partial_{b} X^{\hat{\mu}}=\gamma^{a b} \partial_{b} \tilde{X}_{\hat{\nu}} \mathcal{G}^{\hat{\mu} \hat{\nu}}+\epsilon^{a b} \partial_{b} \mathcal{B}^{\hat{\mu} \hat{\nu}}
$$

In order to examine, how we could expose T-duality from equations of motion, we can rewrite the equation of motion form $X^{\hat{\mu}}$ and $\tilde{X}^{\hat{\mu}}$ in a suitable manner. We multiply the former by $G^{-1}$ and the latter by $\mathcal{G}^{-1}$ to arrive at

$$
\left(G^{-1}\right)^{\hat{\mu} \hat{\nu}} \partial_{a} \tilde{X}_{\hat{\nu}}-\left(G^{-1} B\right)_{\hat{\nu}}^{\hat{\mu}} \partial_{a} X^{\hat{\nu}}=\epsilon_{a}^{b} \partial_{b} X^{\hat{\mu}}
$$

and

$$
\left(\mathcal{G}^{-1}\right)_{\hat{\mu} \hat{\nu}} \partial_{a} X^{\hat{\nu}}-\left(\mathcal{G}^{-1} \mathcal{B}\right)_{\hat{\mu}}^{\hat{\nu}} \partial_{a} \tilde{X}_{\hat{\nu}}=\epsilon_{a}^{b} \partial_{b} \tilde{X}_{\text {hat } \mu}
$$

Note that these are the two currents whose diverges vanish. Now define $\bar{Z}^{i}=$ $\left\{X^{\hat{\mu}}, \bar{X}_{\hat{\mu}}\right\}$ which is analogous to $Z=\left(P, X^{\prime}\right)$ which appeared in the definition of the canonical Hamiltonian density. The above two equations can be combined to a single equation

$$
M \eta \partial_{a} \bar{Z}=\epsilon_{a}^{b} \bar{Z}
$$


where $M$ is defined in terms of constant backgrounds $G$ and $B$. Obviously, the current is conserved. Moreover, the equation is $O(\hat{D}, \hat{D})$ covariant. Therefore, we have cast the equations of motion in a T-duality covariant form once we introduced the dual coordinates and corresponding dual backgrounds. If the string coordinates are defined on compactified target manifold, i.e. the coordinates are periodic, then the dual coordinates will satisfy the same periodicity conditions. As discussed earlier, we generally deal with situations where the target manifold is compactified such that some of the spatial dimensions are quite small and compact. Under such a circumstance, we are unable to probe them. Therefore, in the low energy regime, we assume that the background fields (generally massless excitations of the string) are independent of these coordinates. The simplest compactification scheme is to assume that the internal space is a torus. The toroidal compactification might not be the best choice for string theory when one is attempting to demonstrate emergence of the standard model of particle physics from string theoretic effective action. However, T-duality plays a very important role in string theory. Therefore, consequences of toroidal compactification and underlying symmetries of string theories in this scheme have been studied very extensively over couple of decades.

\subsection{Toroidal Compactication and Symmetry of Evolution Equations}

We continue with our discussion of T-duality symmetry from the worldsheet point of view. The next step is to envisage the scenario where we decompose the string coordinates as follows: $X^{\hat{\mu}}=\left(X^{\mu}, Y^{\alpha}\right)$. Here $X^{\mu}, \mu=0,1,2 \ldots D-1$ are the spacetime coordinates and $Y^{\alpha}, \alpha=D, D+1, \hat{D}-1$ are toroidally compactified coordinates (d of them) so that $D+d=\hat{D}$. Moreover, the $\hat{D}$-dimensional backgrounds $G_{\hat{\mu} \hat{\nu}}$ and $B_{\hat{\mu} \hat{\nu}}$ are independent of $Y^{\alpha}$. Since the spacetime (now) is $D$-dimensional, all the tensors should transform according to the transformation rules of this lower dimensional spacetime. The components of the $\hat{D}$-dimensional tensors lying along compact directions transform as scalars from the point of view of $D$-dimensional spacetime. For example, the $\hat{D}$-dimensional metric decomposes into a symmetric tensor, vectors and scalars (moduli) when the theory is compactified. Scherk and Schwarz [24] have laid down a procedure for decomposition of tensors under general compactification scheme (even when the internal manifold has nontrivial curvature). In the context of toroidal compactification, the procedure becomes relatively simple since torus is flat. Generally, the dimensional reduction scheme is adopted for string effective actions; however, in the context of T-duality for strings in the worldsheet, the tensors are decomposed according to the same prescription. This aspect is not frequently elaborated in literature. Now we make an excursion to this topic.

It is most appropriate to adopt the vielbein formalism for the metric for this purpose $[24]$

$$
e_{\hat{\mu}}^{\hat{r}}=\left(\begin{array}{cc}
e_{\mu}^{r}(X) & A_{\mu}^{(1) \beta}(X) E_{\beta}^{a}(X) \\
0 & E_{\alpha}^{a}(X)
\end{array}\right)
$$


The spacetime metric is $g_{\mu \nu}=e_{\mu}^{r} g_{r s}^{(0)} e_{\nu}^{s}$ and the internal metric is $G_{\alpha \beta}=E_{\alpha}^{a} \delta_{a b} E_{\beta}^{b}$; $g_{r s}^{(0)}$ is the D-dimensional flat space Lorentzian signature metric. $A_{\mu}^{(1) \beta}$ are gauge fields associated with the d-isometries and it is assumes that the backgrounds depend on coordinates $X^{\mu}$ and are independent of $Y^{\alpha}$. Similarly, the antisymmetric tensor background, depending only on $X^{\mu}$ can be decomposed as

$$
B_{\hat{\mu} \hat{\nu}}=\left(\begin{array}{ll}
B_{\mu \nu}(X) & B_{\mu \alpha}(X) \\
B_{\nu \beta}(X) & B_{\alpha \beta}(X)
\end{array}\right)
$$

Here we note the presence of gauge fields $B_{\mu \alpha}$ due to compactification as expected. The worldsheet action (4) will be decomposed into sum of several terms once we adopt the compactification; however, all backgrounds i.e. $g_{\mu \nu}, A_{\mu}^{(1) \alpha}, G_{\alpha \beta}, B_{\mu \nu}, B_{\mu \alpha}$ and $B_{\alpha \beta}$ depend only on spacetime coordinates $X^{\mu}$. In what follows, we closely adopt the technique of [22] to study T-duality symmetry through the evolution equations of string coordinates on the worldsheet. Some charifications are desirable about the notation adopted for background fields. Here $g_{\hat{\mu} \hat{\nu}}$ is the string frame metric in $\hat{D}$ dimensions and $g_{\mu \nu}$ is the metric in $D$-dimensions (defined above). The moduli are $G_{\alpha \beta}$ and $B_{\alpha \beta}$. Note that for constant backgrounds, we deliberately chose the notation $G_{\hat{\mu} \hat{\nu}}$ and $B_{\hat{\mu} \nu}$ to define the $M$-matrix. We shall define, in the present case, the $M$ matrix in terms of $G_{\alpha \beta}$ and $B_{\alpha \beta}$ (see later). The world sheet action is

$$
S=\frac{1}{2} \int d^{2} \sigma\left(g_{\hat{\mu} \hat{\nu}} \gamma^{a b}+B_{\hat{\mu} \hat{\nu}} \epsilon^{a b}\right) \partial_{a} X^{\hat{\mu}} \partial_{b} X^{\hat{\nu}}
$$

Varying this with respect to $X^{\hat{\mu}}(\sigma, \tau)$ gives the classical equation of motion for the string

$$
\begin{aligned}
& \frac{\delta S}{\delta X^{\hat{\mu}}}=-\Gamma_{\hat{\mu} \hat{\nu} \hat{\rho}} \partial^{a} X^{\hat{\nu}} \partial_{a} X^{\hat{\rho}}-g_{\hat{\mu} \hat{\nu}} \partial^{a} \partial_{a} X^{\hat{\nu}} \\
& +\frac{1}{2} \epsilon^{a b}\left(\partial_{\hat{\mu}} B_{\hat{\nu} \hat{\rho}}+\partial_{\hat{\nu}} B_{\hat{\rho} \hat{\mu}}+\partial_{\hat{\rho}} B_{\hat{\mu} \hat{\nu}}\right) \partial_{a} X^{\hat{\nu}} \partial_{b} X^{\hat{\rho}}=0
\end{aligned}
$$

where

$$
\Gamma_{\hat{\mu} \hat{\nu} \hat{\rho}}=\frac{1}{2}\left(\partial_{\hat{\nu}} g_{\hat{\mu} \hat{\rho}}+\partial_{\hat{\rho}} g_{\hat{\mu} \hat{\nu}}-\partial_{\hat{\mu}} g_{\hat{\nu} \hat{\rho}}\right)
$$

With the insight from the case of constant backgrounds where we derived T-duality covariant equations of motion, it is convenient to analyze the equations of motion for $Y^{\alpha}$ separately first. Therefore, we look at the action which depends on $Y$,

$$
S_{Y}=\int d^{2} \sigma\left\{\frac{1}{2}\left(\gamma^{a b} G_{\alpha \beta}(X) \partial_{a} Y^{\alpha} \partial_{b} Y^{\beta}+\epsilon^{a b} B_{\alpha \beta}(X) \partial_{a} Y^{\alpha} \partial_{b} Y^{\beta}\right)+\Gamma_{\alpha}^{a}(X) \partial_{a} Y^{\alpha}\right\}
$$

and

$$
\Gamma_{\alpha}^{a}=\gamma^{a b} G_{\alpha \beta} A_{\mu}^{(1) \beta} \partial_{b} X^{\mu}-\epsilon^{a b}\left(A_{\mu \alpha}^{(2)}-B_{\alpha \beta} A_{\mu}^{(1) \beta}\right) \partial_{b} X^{\mu}
$$


encodes information about the gauge fields $A_{\mu}^{(1) \alpha}$ and $A_{\mu \alpha}^{(2)}$. This action generalizes earlier equations, both by including background vector fields and by allowing $X$ dependence for all the background fields. Our aim is to study the equations of motion of $Y$ and suitably modify them so that these equations are manifestly $O(d, d)$ covariant. Thus we have to introduce dual coordinates and corresponding backgrounds for the case at hand. Intuitively we can see that when we consider strings in flat backgrounds, i.e. massless backgrounds are trivial $Y$ and $\tilde{Y}$ would correspond to the sum and difference of left-moving and right-moving components. In more general settings, the interpretation is not quite so simple.

Since the backgrounds are independent of $Y^{\alpha}$, the Euler-Lagrange equations take the form

$$
\partial_{a}\left(\frac{\delta S}{\delta \partial_{a} Y^{\alpha}}\right)=0
$$

Therefore, locally, we can write

$$
\frac{\delta S}{\delta \partial_{a} Y^{\alpha}}=\gamma^{a b} \partial_{b} Y^{\beta} G_{\alpha \beta}+\epsilon^{a b} \partial_{b} Y^{\beta} B_{\alpha \beta}+\Gamma_{\alpha}^{a}=\epsilon^{a b} \partial_{b} \tilde{Y}_{\alpha}
$$

where $\tilde{Y}_{\alpha}$ are the dual coordinates as before. They will have the same periodicities as the $Y^{\alpha}$. Introducing auxiliary fields $U_{a}^{\alpha}$, let us now define a dual action for the case at hand

$$
\tilde{S}=\int d^{2} \sigma\left\{\frac{1}{2}\left(\gamma^{a b} U_{a}^{\alpha} U_{b}^{\beta} G_{\alpha \beta}+\epsilon^{a b} U_{a}^{\alpha} U_{b}^{\beta} B_{\alpha \beta}\right)+\epsilon^{a b} \partial_{a} \tilde{Y}_{\alpha} U_{b}^{\alpha}+\Gamma_{\alpha}^{a} U_{a}^{\alpha}\right\}
$$

If we vary this action with respect to $\tilde{Y}_{\alpha}$, we get $\partial_{a}\left(\epsilon^{a b} U_{b}^{\alpha}\right)=0$. The auxiliary field equation, now more complicated, becomes

$$
\eta^{a b} U_{b}^{\beta} G_{\alpha \beta}+\epsilon^{a b} U_{b}^{\beta} B_{\alpha \beta}-\epsilon^{a b} \partial_{b} \tilde{Y}_{\alpha}+\Gamma_{\alpha}^{a}=0
$$

agrees with equation of motion of $Y^{\alpha}$ when we identify $U_{a}^{\alpha}$ with $\partial_{a} Y^{\alpha}$. Although, more complicated compared to constant $G$ and $B$ case, we can solve for for $U_{a}^{\alpha}$ in terms of $\partial_{a} \tilde{Y}_{\alpha}$ and $\Gamma_{\alpha}^{a}$ and arrive at

$$
U_{a}^{\alpha}=\left(\epsilon_{a}^{b} \mathcal{G}^{\alpha \beta}+\delta_{a}^{b} \mathcal{B}^{\alpha \beta}\right)\left(\partial_{b} \tilde{Y}_{\beta}-\epsilon_{b c} \Gamma_{\beta}^{c}\right)
$$

As before

$$
\mathcal{G}=\left(G-B G^{-1} B\right)^{-1}
$$

and

$$
\mathcal{B}=-G^{-1} B\left(G-B G^{-1} B\right)^{-1}
$$

Note that (i) the backgrounds depend on string coordinates $X^{\mu}$ and (ii) $(G+B)(\mathcal{G}+$ $\mathcal{B})=1$, so that $\mathcal{G}$ and $\mathcal{B}$ are the symmetric and antisymmetric parts of $(G+B)^{-1}$, 
respectively. If we substitute for $U_{a}^{\alpha}$ in dual action, we arrive at

$$
\begin{aligned}
\tilde{S}=\quad & \int d^{2} \sigma\left\{\frac{1}{2}\left(\gamma^{a b} \partial_{a} \tilde{Y}_{\alpha} \partial_{b} \tilde{Y}_{\beta} \mathcal{G}^{\alpha \beta}+\epsilon^{a b} \partial_{a} \tilde{Y}_{\alpha} \partial_{b} \tilde{Y}_{\beta} \mathcal{B}^{\alpha \beta}\right)-\epsilon_{b}^{a} \partial_{a} \tilde{Y}_{\alpha} \Gamma_{\beta}^{b} \mathcal{G}^{\alpha \beta}\right. \\
& \left.-\partial_{a} \tilde{Y}_{\alpha} \Gamma_{\beta}^{a} \mathcal{B}^{\alpha \beta}-\frac{1}{2}\left(\gamma_{a b} \Gamma_{\alpha}^{a} \Gamma_{\beta}^{b} \mathcal{G}^{\alpha \beta}+\epsilon_{a b} \Gamma_{\alpha}^{a} \Gamma_{\beta}^{b} \mathcal{B}^{\alpha \beta}\right)\right\}
\end{aligned}
$$

Following remarks deserve mention (i) $\mathcal{G}^{\alpha \beta}$ and $\mathcal{B}^{\alpha \beta}$ are determined in terms of $G_{\alpha \beta}$ and $B_{\alpha \beta}$, (ii) they depend only on $X^{\mu}$, (iii) so does $\Gamma_{\alpha}^{a}$. Moreover, the equation of motion derived from $\tilde{S}$ is

$$
\partial_{a}\left(\frac{\delta \tilde{S}}{\delta \partial_{a} \tilde{Y}_{\alpha}}\right)=0
$$

The two Lagrangians $S$ and $\tilde{S}$ give a pair of equivalent equations of motion (at least locally)

$$
\epsilon^{a b} \partial_{b} Y^{\alpha}=\frac{\delta \tilde{S}}{\delta \partial_{a} \tilde{Y}_{\alpha}}=\gamma^{a b} \partial_{b} \tilde{Y}_{\beta} \mathcal{G}^{\alpha \beta}+\epsilon^{a b} \partial_{b} \tilde{Y}_{\beta} \mathcal{B}^{\alpha \beta}-\epsilon_{b}^{a} \mathcal{G}^{\alpha \beta} \Gamma_{\beta}^{b}-\mathcal{B}^{\alpha \beta} \Gamma_{\beta}^{a}
$$

In order to express an equation in an $O(d, d)$ covariant form, we have to combine the pair of equations of motion derived for $Y^{\alpha}$ and $\tilde{Y}^{\alpha}$ in suitable manner as was done for constant backgrounds. Although these equations are a lot more complicated, this goal can be achieved. The pair of equations are

$$
\begin{gathered}
G^{\alpha \beta} \partial_{a} \tilde{Y}_{\beta}-\left(G^{-1} B\right)^{\alpha}{ }_{\beta} \partial_{a} Y^{\beta}=\epsilon_{a}{ }^{b} \partial_{b} Y^{\alpha}+\epsilon_{a b} G^{\alpha \beta} \Gamma_{\beta}^{b} \\
\left(\mathcal{G}^{-1}\right)_{\alpha \beta} \partial_{a} Y^{\beta}-\left(\mathcal{G}^{-1} \mathcal{B}\right)_{\alpha}{ }^{\beta} \partial_{a} \tilde{Y}_{\beta}=\epsilon_{a}{ }^{b} \partial_{b} \tilde{Y}_{\alpha}-\eta_{a b} \Gamma_{\alpha}^{b}-\epsilon_{a b}\left(\mathcal{G}^{-1} \mathcal{B}\right)_{\alpha}{ }^{\beta} \Gamma_{\beta}^{b}
\end{gathered}
$$

We define the enlarged manifold by combining the compact coordinates and their corresponding dual coordinates like the previous case. We are guided by the intuition that the equations of motion for the present case are still conservation laws (for compact coordinates) although the worldsheet action for $Y^{\alpha}$ and $\tilde{Y}^{\alpha}$ are lot more complex. Defining $\left\{\tilde{Z}^{i}\right\}=\left\{Y^{\alpha}, \tilde{Y}_{\alpha}\right\}, i=1,2, \ldots, 2 d$, then the above two equations are combined to a single equation can be combined as the single equation

$$
M \eta \partial_{a} Z=\epsilon_{a}^{b} \partial_{b} \tilde{Z}+M \eta \Sigma_{a}
$$

Here $\Sigma_{a}$ is an $O(d, d)$ vector (for each value of $a$ ) given by the column vector

$$
\Sigma_{a}^{i}=\left(\begin{array}{c}
-\gamma_{a b} G^{\alpha \beta} \Gamma_{\beta}^{b} \\
\epsilon_{a b} \Gamma_{\alpha}^{b}-\eta_{a b} B_{\alpha \gamma} G^{\gamma \beta} \Gamma_{\beta}^{b}
\end{array}\right)
$$

Note that $\Sigma_{a}$ can also re-expressed as

$$
\Sigma_{a}^{i}=-\partial_{a} X^{\mu} \mathcal{A}_{\mu}^{i}+\epsilon_{a}{ }^{b} \partial_{b} X^{\mu}\left(M \eta \mathcal{A}_{\mu}\right)^{i}
$$


where $\mathcal{A}_{\mu}^{i}$ is comprised of $A_{\mu}^{(1) \alpha}$ and $A_{\mu \alpha}^{(2)}$. The former is associated with the $d$ isometries due to compactification, coming from the metric and the latter are the gauge fields as we reduce the two form potential to lower dimensions. Thus we arrive at the first-order equation

$$
M \eta\left(\partial_{a} Z+\mathcal{A}_{\mu} \partial_{a} X^{\mu}\right)=\epsilon_{a}^{b}\left(\partial_{b} Z+\mathcal{A}_{\mu} \partial_{b} X^{\mu}\right)
$$

This is the desired result. However, we have to still deal with the equations of motion associated with $X^{\mu}$. Although, these are genuine equations of motion in the sense that these are not conservation laws due to nontrivial $X^{\mu}$ dependence carried by all backgrounds, it is important to note that any transformation carried out along compact directions do not affect the spacetime tensors and coordinates $X^{\mu}$. Only the moduli and the gauge field (appearing after dimensional reduction) under go transformations under T-duality. However, after some careful manipulations, it can be shown that these equations are $O(d, d)$ invariant.

Now consider the case, when all the string coordinates are compactified on $\hat{D}$-dimensional torus, $T^{\hat{D}}$. We denote all these coordinates as $Y^{\alpha}$. They satisfy the condition

$$
Y^{\alpha}(\sigma, \tau)+2 \pi=Y^{\alpha}(\sigma, \tau)
$$

compactification radii is 1 and the string is still in constant backgrounds. To distinguish from noncompact coordinates, we have denoted compact coordinates as $Y^{\alpha}, Y^{\beta}, \alpha, \beta=0,1, \hat{D}$; this is a special case of compactification scheme we have just discussed. However, we intend to illustrate how the discrete symmetry $O(d, d ; Z)$ appears from our perspective. Moreover, just for our conveniences, we take spacetime target space metric to be of Euclidean signature for this particular example. If we consider compactification of some of the spatial coordinates on $T^{d}$ such that

$$
X^{\hat{\mu}}=\left(X^{\mu}, Y^{\alpha}\right)
$$

and $Y^{\alpha}$ are compact coordinates then the metric on $T^{d}$ is indeed Euclidean. However, when we leave some coordinates uncompactified, we shall always consider unusual Lorenzian signature. Consider motion of a particle on a circular path. The momentum is quantized in suitable units of the inverse radius in order that the wave function is single valued. Next we consider a massless scalar field, $\phi$ in $\hat{D}$ dimensions with coordinates $x^{\hat{\mu}}$; however, we assume that one of its spatial coordinates is compact, $S^{1}$ with radius, $R$. As is well known, the lower dimensional theory, when $R$ is small has a spectrum consisting of a massless scalar and a tower of massive states with a spectrum $\frac{n^{2}}{R^{2}}$. This is the Kaluza-Klein compactification. However, in case of a string, one of whose coordinate has geometry of a circle, offers more interesting possibilities. A distinctive feature of string theory, with such a compactification is: we cannot distinguish the perturbative spectrum of this theory (compactification radius $R$ ) from that of another string theory whose coordinate is compactified on a circle of radius 
$\frac{1}{R}$. The reason is that the (closed) string compact coordinate also satisfies periodic boundary condition and this coordinate can wind around the circle;

$$
Y(\sigma, \tau)+2 \pi R=Y(\sigma, \tau)
$$

Furthermore, the string coordinate is also periodic when $\sigma$ goes over $2 \pi$ for the closed string. Since, the coordinate is compact, zero momentum mode must be quantized to maintain single valuedness of the wave function just as the case in field theory. In case of the string, the string can wind around the compact direction. It will cost more energy if the string winds m-number time, because it will have to stretch more. Therefore, the effect due to windings has to be taken into account too while estimating energy levels.

$$
\begin{aligned}
& Y_{R}=y_{R}+\sqrt{\frac{1}{2}} p_{R}(\tau-\sigma)+\text { oscillators } \\
& Y_{L}=y_{L}+\sqrt{\frac{1}{2}} p_{L}(\tau+\sigma)+\text { oscillators }
\end{aligned}
$$

The momentum zero modes $p_{R, L}$ will have the following form to be consistent with what we said earlier

$$
p_{R}=\frac{1}{\sqrt{2}}\left(\frac{n}{R}-R m\right), \text { and } p_{L}=\frac{1}{\sqrt{2}}\left(\frac{n}{r}+R m\right)
$$

Here we have displayed the presence of the compactification radius, $R$, which is generally set to unity. It is explicitly displayed to demonstrate $R \rightarrow \frac{1}{R}$ T-duality which interchanges $\mathrm{K}-\mathrm{K}$ modes and winding modes. The above equation states that in general the contribution of the Kaluza-Klein mode is $\frac{1}{R}$ times an integer and the winding mode is an integer times the radius. The total momentum is just $P=\frac{1}{\sqrt{2}}\left(p_{R}+p_{L}\right)$, which is integral of momentum density over $\sigma$. The total Hamiltonian is

$$
H=L_{0}+\bar{L}_{0}=\frac{1}{2}\left(p_{L}^{2}+p_{R}^{2}\right)+\text { oscillators }
$$

We can generalize the above argument for the case of a closed string with compact coordinates $Y^{\alpha}(\tau, \sigma)$ in the presence constant massless backgrounds $G_{\alpha \beta}$ and $B_{\alpha \beta}$. Now we consider the general case of toroidal compactification and present the derivation as was done in reference [22]. Let $G_{\alpha \beta}$ and $B_{\alpha \beta}$ be constant backgrounds, $\alpha, \beta=$ $1, \ldots d$, and $Y^{\alpha}(\sigma, \tau)$ are the string coordinates. Here $d=\hat{D}$ since we take all coordinates to be compact. The two-dimensional $\sigma$-model The two-dimensional $\sigma$-model action containing these coordinates is

$$
S_{\text {compact }}=\frac{1}{2} \int d^{2} \sigma\left[G_{\alpha \beta} \eta^{a b} \partial_{a} Y^{\alpha} \partial_{b} Y^{\beta}+\epsilon^{a b} B_{\alpha \beta} \partial_{a} Y^{\alpha} \partial_{b} Y^{\beta}\right]
$$

where $G_{\alpha \beta}$ and $B_{\alpha \beta}$ are constant backgrounds. The coordinates are taken to satisfy the periodicity conditions $Y^{\alpha} \simeq Y^{\alpha}+2 \pi$. Here we take the compactification radius 
to be unity i.e. $R=1$, for simplicity in calculations. For closed strings it is necessary that

$$
Y^{\alpha}(2 \pi, \tau)=Y^{\alpha}(0, \tau)+2 \pi m^{\alpha}
$$

where the integers $m^{\alpha}$ are called winding numbers. It follows from the singlevaluedness of the wave function on the torus that the zero modes of the canonical momentum, $P_{\alpha}=G_{\alpha \beta} \partial_{\tau} Y^{\beta}+B_{\alpha \beta} \partial_{\sigma} Y^{\beta}$, are also integers $n_{\alpha}$. Therefore the zero modes of $Y^{\alpha}$ are given by

$$
Y_{0}^{\alpha}=y^{\alpha}+m^{\alpha} \sigma+G^{\alpha \beta}\left(n_{\beta}-B_{\beta \gamma} n^{\gamma}\right) \tau
$$

where $G^{\alpha \beta}$ is the inverse of $G_{\alpha \beta}$. The Hamiltonian is given by

$$
\mathcal{H}=\frac{1}{2} G_{\alpha \beta}\left(\dot{Y}^{\alpha} \dot{Y}^{\beta}+Y^{\prime \alpha} Y^{\prime \beta}\right)
$$

where $\dot{Y}^{\alpha}$ and $Y^{\prime \beta}$ are derivatives with respect to $\tau$ and $\sigma$, respectively.

Since $Y^{\alpha}(\sigma, \tau)$ satisfies the free wave equation, we can decompose it as the sum of left- and right-moving pieces. The zero mode of $P^{\alpha}=G^{\alpha \beta} P_{\beta}$ is given by $p_{L}^{\alpha}+p_{R}^{\alpha}$ where

$$
\begin{aligned}
p_{L}^{\alpha} & =\frac{1}{2}\left[m^{\alpha}+G^{\alpha \beta}\left(n_{\beta}-B_{\beta \gamma} m^{\gamma}\right)\right] \\
p_{R}^{\alpha} & =\frac{1}{2}\left[-m^{\alpha}+G^{\alpha \beta}\left(n_{\beta}-B_{\beta \gamma} m^{\gamma}\right)\right]
\end{aligned}
$$

The mass-squared operator, which corresponds to the zero mode of $\mathcal{H}$, is given (aside from a constant) by

$$
(m a s s)^{2}=G_{\alpha \beta}\left(p_{L}^{\alpha} p_{L}^{\beta}+p_{R}^{\alpha} p_{R}^{\beta}\right)+\sum_{m=1}^{\infty} \sum_{i=1}^{d}\left(\alpha_{-m}^{i} \alpha_{m}^{i}+\bar{\alpha}_{-m}^{i} \bar{\alpha}_{m}^{i}\right)
$$

As usual, $\left\{\alpha_{m}\right\}$ and $\left\{\bar{\alpha}_{m}\right\}$ denote oscillators associated with right- and left-moving coordinates, respectively. Substituting the expressions for $p_{L}$ and $p_{R}$, the mass squared can be rewritten as

$$
(m a s s)^{2}=\frac{1}{2} G_{\alpha \beta} m^{\alpha} m^{\beta}+\frac{1}{2} G^{\alpha \beta}\left(n_{\alpha}-B_{\alpha \gamma} m^{\gamma}\right)\left(n_{\beta}-B_{\beta \delta} m^{\delta}\right)+\sum\left(\alpha_{-m}^{i} \alpha_{m}^{i}+\bar{\alpha}_{-m}^{i} \bar{\alpha}_{m}^{i}\right)
$$

It is significant that the zero mode portion of (60) can be expressed in the form

$$
\left(M_{0}\right)^{2}=\frac{1}{2}\left(\begin{array}{ll}
m & n
\end{array}\right) M^{-1}\left(\begin{array}{c}
m \\
n
\end{array}\right)
$$

where $M$ is the $2 d \times 2 d$ symmetric matrix expressed in terms of constant backgrounds $G$ and $B$

$$
M=\left(\begin{array}{cc}
G^{-1} & -G^{-1} B \\
B G^{-1} & G-B G^{-1} B
\end{array}\right)
$$


In order to satisfy $\sigma$-translation symmetry, the contributions of left- and right-moving sectors to the mass squared must agree; $L_{0}=\bar{L}_{0}$. The zero mode contribution to their difference is

$$
G_{\alpha \beta}\left(p_{L}^{\alpha} p_{L}^{\beta}-p_{R}^{\alpha} p_{R}^{\beta}\right)=m^{\alpha} n_{\alpha}
$$

Since this is an integer, it always can be compensated by oscillator contributions, which are also integers.

Equation (63) is invariant under interchange of the winding numbers $m^{\alpha}$ and the discrete momenta $n_{\alpha}$. Indeed, the entire spectrum remains invariant if we interchange $m^{\alpha} \leftrightarrow n_{\alpha}$ simultaneously let [22]

$$
\left(G-B G^{-1} B\right) \leftrightarrow G^{-1} \quad \text { and } \quad B G^{-1} \leftrightarrow-G^{-1} B
$$

These interchanges precisely correspond to inverting the $2 d \times 2 d$ matrix $M$. This is the spacetime duality transformation generalizing the well-known duality $R \leftrightarrow \frac{1}{R}$ in the $d=1$ case discussed earlier. The general duality symmetry implies that the $2 d$ dimensional Lorentzian lattice spanned by the vectors $\sqrt{2}\left(p_{L}^{\alpha}, p_{R}^{\alpha}\right)$ with inner product

$$
\sqrt{2}\left(p_{L}, p_{R}\right) \cdot \sqrt{2}\left(p_{L}^{\prime}, p_{R}^{\prime}\right) \equiv 2 G_{\alpha \beta}\left(p_{L}^{\alpha} p_{L}^{\prime \beta}-p_{R}^{\alpha} p_{R}^{\prime \beta}\right)=\left(m^{\alpha} n_{\alpha}^{\prime}+m^{\prime \alpha} n_{\alpha}\right)
$$

is even and self-dual ([30]).

The moduli space parametrized by $G_{\alpha \beta}$ and $B_{\alpha \beta}$ is locally the coset $O(d, d) / O(d) \times$ $O(d)$. The global geometry requires also modding out the group of discrete symmetries generated by $B_{\alpha \beta} \rightarrow B_{\alpha \beta}+N_{\alpha \beta}$ and $G+B \rightarrow(G+B)^{-1}$. These symmetries generate the $O(d, d, Z)$ subgroup of $O(d, d)$. An $O(d, d, Z)$ transformation is given by a $2 d \times 2 d$ matrix $A$ having integral entries and satisfying $A^{T} \eta A=\eta$, where $\eta$ consists of offdiagonal unit matrices defined below. Under an $O(d, d, Z)$ transformation

$$
\left(\begin{array}{c}
m \\
n
\end{array}\right) \rightarrow\left(\begin{array}{c}
m^{\prime} \\
n^{\prime}
\end{array}\right)=A\left(\begin{array}{c}
m \\
n
\end{array}\right) \quad \text { and } \quad M \rightarrow A M A^{T}
$$

It is evident that

$$
\begin{gathered}
m \cdot n=\frac{1}{2}\left(\begin{array}{ll}
m & n
\end{array}\right) \eta\left(\begin{array}{c}
m \\
n
\end{array}\right) \\
\eta=\left(\begin{array}{ll}
0 & \mathbf{1} \\
\mathbf{1} & 0
\end{array}\right),
\end{gathered}
$$

which appears in eq.(63), and $M_{0}^{2}$ in eq.(60) are preserved under these transformations. Note that $\eta$ is symmetric $2 d \times 2 d$ matrix with off diagonal elements which are d-dimensional unit matrices. The crucial fact, already evident from the spectrum, is that toroidally compactified string theory certainly does not share the full $O(d, d)$ symmetry of the low energy effective theory. It is at most invariant under the discrete $O(d, d, Z)$ subgroup.

Let us very briefly discuss the role of T-duality symmetry in open string theories and we focus on bosonic string theory in order to illustrate the salient features. When 
we consider toroidal compactification of a closed string and examine its spectrum, we discover that both $\mathrm{K}-\mathrm{K}$ modes and winding modes contribute to the spectrum besides the excitations due to the action of oscillators on the vacuum. Since open string has no analog of winding modes one might think that T-duality has no important roles for open string theories. However, the open string admits both Dirichlet and Neumann boundary conditions when we look for solutions to equations of motion. In recent years, it is recognized that $D_{p}$ branes play a very important role in our understanding of string dynamics. We visualize the situation as follows. These are solitonic objects and they have conformal field theory descriptions. In $D$-dimensions, if there is a $D_{p}$-brane, there are Neumann boundary conditions satisfied in $(p+1)$ diections. These are directions of the worldvolume coordinates of $D_{p}$-brane and we have Dirichlet boundary conditions along the remaining transverse directions that is $(D-p-1)$ coordinates satisfy Dirichlet boundary conditions. Thus open strings can have their end points stuck to these hypersurfances and oscillate. Put more explicitly, the boundary conditions are as follows

$$
\partial_{\sigma} X^{\mu}=0, \text { for } \mu=0,1, . . p-1
$$

are the Neumann boundary conditions and

$$
X^{\mu}(\sigma=0, \pi)=a_{0}^{\mu}, \text { for } \mu=p, p+1 . . D-1
$$

correspond to Dirichlet boundary conditions. A $D_{p}$-brane will couple to $p+2$-form $\mathrm{RR}$ field strength. Therefore, $D_{0}$-brane is interpreted as a particle, $D_{1}$-brane is identified as a D-string and so on. Note that translational invariance is broken along $\mu=$ $p, . . D-1$ and superstring $D=10$. Recall that T-duality along a give direction can take to a Neumann boundary condition to Dirichlet or vice versa. Thus a $D_{p}$-brane can be converted to a $D_{p+1}$-brane or a $D_{p-1}$-brane as we desire. We elucidated, in nutshell, how T-duality can be utilized in the context open strings. Open strings oscillate in $d$-dimensions while their end points are fixed on a $p+1$-dimensional hyperplane and we call it $D_{p}$ brane. Thus the open strings whose end points are fixed on these hypersurfaces satisfy Dirichlet boundary conditions in the $d-p-1$ transverse directions. However, in those directions, one looses translational invariance. We have alluded earlier that T-duality along a given direction is equivalent to $\sigma \leftrightarrow \tau$. Thus, in the context of open string theory, T-duality operation interchanges Neumann and Dirichlet boundary conditions. Therefore, in the context of open string theory, when T-duality is implemented judiciously, one can take a $D_{p}$ brane to $D_{p+1}$ brane or $D_{p-1}$ brane. In other words, we are able to realize various types of brane solutions via T-duality operation as per our requirements.

\subsection{Dimensional Reduction of Effective Action and T-duality}

So far, in discussing issue compactifications, we have considered situations when all the coordinates are compact. However, one can envisage the scenario, when some of 
the string string coordinates are compactified and the rest are noncompact. Furthermore, we treated the backgrounds to be constant; however, in more realistic situations the backgrounds should be allowed to depend on noncompact coordinates. This is the more interesting situation where we have a ten dimensional string theory and six of its spatial coordinates are compactified on a torus $T^{6}$ so that the resulting theory is reduced to a four dimensional effective theory. We shall adopt the general prescription of dimensional reduction $[24,22,58]$ so that we can compactify an arbitrary number of dimensions so that the effective theory is defined in a lower spacetime dimension, not necessarily four. This will be useful, since the duality conjectures are in various spacetime dimensions and string theories are related by the web of dualities in diverse dimensions.

The starting point is to consider the string effective action in $\hat{D}$ spacetime dimensions. The coordinates, metric and all other tensors in the $\hat{D}$ dimensional space are specified with a 'hat'. The coordinates in D-dimensional spacetime are denoted by $x^{\mu}, \mu, \nu, e t c$ are spacetime indices. We discuss a few related points before closing discussions in this section. When evolution of the closed string is envisaged in the background of its massless excitations the worldsheet action assumes the form of a nonlinear $\sigma$-model action. If we impose the constraints of conformal invariance, these massless backgrounds are required to satisfy the so called equations of motion since the associated $\beta$-functions must vanish. If we reconstruct, from these equations of motion, an action in $\hat{D}$-dimensional target space such that the Euler-Langrange equation derived from this action coincides with the equations of motions obtained from the $\beta$-function equations. Therefore, the $\hat{D}$-dimensional 'effective' action is derived order by order in the $\sigma$-model approach since the $\beta$-function equations are derived perturbatively from the two dimensional $\sigma$-model. Moreover, generally, one confines to tree level computation in string perturbation theory in the sense that the $\beta$-function is computed for the lowest genus Riemann surface. We write down the tree level string effective action for the closed string.

$$
S_{e f f}=\int d^{\hat{D}} x \sqrt{-\hat{g}} e^{-\hat{\Phi}}\left(R_{\hat{g}}+(\partial \hat{\Phi})^{2}-\frac{1}{12} H_{\hat{\mu} \hat{\nu} \hat{\rho}} H^{\hat{\mu} \hat{\nu} \hat{\rho}}\right)
$$

where $H_{\hat{\mu} \hat{\nu} \hat{\rho}}=\partial_{\hat{\mu}} B_{\hat{\nu} \hat{\rho}}+$ cyclic perm. $\hat{g}=\operatorname{det} g_{\hat{\mu} \hat{\nu}}$ and $\hat{\Phi}$ are the 10-dimensional string frame metric and dilaton respectively. If we are to compactify the theory, toroidally, to $D$-dimensional spacetime, the fields appearing in definition of $R_{\hat{g}}$ and $H_{\hat{\mu} \hat{\nu} \hat{\rho}}$ we have define resulting fields in lower dimensions appropriately. We have already alluded to the procedure of decomposing the 10-dimensional metric and 2-form fields in terms of fields in lower dimension. From D-dimensional point of view these tensors are decomposed to the metric and 2-form, associated gauge fields, $\left(A_{\mu}^{(1) \alpha}, A_{\mu \alpha}^{(2)}\right)$ and the moduli, $\left(G_{\alpha \beta}, B_{\alpha \beta}\right)$. All these fields are independent of the compact coordinates $y^{\alpha}$. The next step is to express all the reduced tensors and vectors in such a way that that their transformations under general coordinate transformations confirm with those of 
D-dimensional spacetime. Thus $S_{\text {eff }}$ is dimensionally reduced to [24, 22]

$$
S_{e f f}=S_{1}+S_{2}+S_{3}
$$

where

$$
\begin{gathered}
S_{1}=\int d^{D} x \sqrt{-} g e^{-\phi}\left(R_{g}+(\partial \phi)^{2}-\frac{1}{12} H_{\mu \nu \rho} H^{\mu \nu \rho}\right) \\
S_{2}=-\frac{1}{4} \int d^{D} x \sqrt{-} g e^{-\phi} \mathcal{F}_{\mu \nu}^{T} \mathcal{F}^{\mu \nu} \\
S_{3}=\frac{1}{8} \int d^{D} x \sqrt{-} g e^{-\phi} \partial_{\mu} M^{-1} \partial^{\mu} M
\end{gathered}
$$

Note that $R_{g}$ is the scalar curvature computed from the D-dimensional metric $g_{\mu \nu}$. $\phi=\hat{\Phi}-\frac{1}{2} \log \operatorname{det} G_{\alpha \beta}$ is the shifted dilaton. $H_{\mu \nu \rho}$ is defined as follows:

$$
H_{\mu \nu \rho}=\partial_{\mu} B_{\nu \rho}-\frac{1}{2}\left(A^{(1) \alpha} F_{\nu \rho \alpha}^{(2)}+A_{\mu \alpha}^{(2)} F_{\nu \rho}^{(1) \alpha}\right)+\text { cyc.perm }
$$

with $B_{\mu \nu}$ defined as

$$
B_{\mu \nu}=\hat{B}_{\mu \nu}+\frac{1}{2} A_{\mu}^{(1) \alpha} A_{\nu \alpha}^{(2)}-\frac{1}{2} A_{\nu}^{(1) \alpha} A_{\mu \alpha}^{(2)}-A_{\mu}^{(1) \alpha} B_{a l p h a \beta} A^{(1) \beta}
$$

We define $\hat{B}_{\mu \nu}$ to be $\mu, \nu$ component of $\hat{D}$-dimensional antisymmetric tensor. The last three terms arise due to dimensional reduction [22]. The gauge field strengths $\mathcal{F}_{\mu \nu}$ are defined as follows which transform as $O(d, d)$ vectors. and

$$
\mathcal{F}_{\mu \nu}=\left(\begin{array}{c}
\partial_{\mu} A_{\nu}^{(1) \alpha}-\partial_{\nu} A_{\mu}^{(1) \alpha} \\
\partial_{\mu} A_{\alpha \nu}^{(2)}-\partial_{\nu} A_{\alpha \mu}^{(2)}
\end{array}\right)
$$

and

$$
M=\left(\begin{array}{cc}
G^{-1} & -G^{-1} B \\
B G^{-1} & G-B G^{-1} B
\end{array}\right)
$$

is the $M$-matrix defined earlier which depends on spacetime coordinate $x^{\mu}$. Note that each of the terms $S_{1}, S_{2}$ and $S_{3}$ are $O(d, d)$ invariant on their own. The $O(d, d)$ invariance of the effective action has played a very important role in generating new solutions from a given set of backgrounds which follow from equations of motion. In other words, if we have a set of backgrounds as solutions to $\beta$-function equations ("equations of motion") by implementing $O(d, d)$ transformations judiciously, we can generate new configurations which are also solutions to equations of equations. Therefore, we can go from one string vacuum to another one which is not connected to the former through any gauge transformation i.e. general coordinate transformation, gauge transformation of $A_{\mu}^{(1) \alpha}$ or $A_{\mu \alpha}^{(2)}$ or gauge transformation associated with two form $B_{\mu \nu}$. 
We present a very simple example to illustrate how $O(d, d)$ symmetry is utilized for generating new solutions starting from a solution of equation of motion. Consider the cosmological scenario where all backgrounds depend on the cosmic time $t$. We can write

$$
\begin{aligned}
& g_{\hat{\mu} \hat{\nu}}=\left(\begin{array}{cc}
1 & 0 \\
0 & G_{i j}
\end{array}\right) \\
& B_{\hat{\mu} \hat{\nu}}=\left(\begin{array}{cc}
0 & 0 \\
0 & B_{i j}
\end{array}\right)
\end{aligned}
$$

Note that, for this case we can always bring the metric $g_{\hat{\mu} \hat{\nu}}$ to this form using a general coordinate transformation and $G_{i j}, i, j=1, . . \hat{D}-1$ is the spatial part of the metric - can be identifies with $G_{\alpha \beta}$. Similarly, $B_{\hat{\mu} \hat{\nu}}$ also can be brought to the present form using the gauge transformation on the 2 -form $\mathrm{B}$. Its $t-t$ component vanishes from the antisymmetry property. The shifted dilaton on this occasion is $\phi=\hat{\Phi}-\frac{1}{2} \log \operatorname{det} G_{i j}$. One can start from a cosmological solution where $G$ and $\phi$ are nontrivial. Then implement an appropriate global $O(D, D)$ transformation (that is the duality group here) and generate a new cosmological solution where we have $\tilde{G}, \tilde{B}$ and $\phi$ such that

$G$ and $\tilde{G}$ are not related by general coordinate transformation. Moreover, $B$ and $\tilde{B}$ are not connected by the 'vector' gauge transformation of 2 -form. The shifted dilaton remains invariant as is the case with $O(D, D)$ transformation (see $[7,54]$ for more details).

\section{Massive Excited States and T-duality}

In this section we explore duality symmetry associated with massive excited stated of closed string from the worldsheet view point. The evolution of the string in the background of its massless excitation corresponds to a 2-dimensional $\sigma$-model where the backgrounds are identified as coupling constants of the theory. These are constrained if we demand that the theory respects conformal invariance. We intend to follow a similar approach where the string evolves in the background of higher massive levels in order to study the duality symmetry associated with the excited states. It is recognized that excited massive stringy states have many interesting roles in string theory.

Indeed a close examination of string propagation in its massless backgrounds reveals the importance of excited massive levels in string theory. Let us follow the arguments put forward by Das and Sathiapalan [59, 60]. In order to investigate implications of conformal invariance, we envisage the $\sigma$-model in the weak field approximation. To be specific, consider a closed bosonic string in the graviton background. For sake of simplicity, when we resort to weak field approximation we write $g_{\mu \nu}=g_{\mu \nu}^{0}+h_{\mu \nu}$ where $g_{\mu \nu}^{0}$ is the flat space Lorentzian metric and $h_{\mu \nu}$ is the fluctuation (i.e. graviton). 
Moreover, we expand the string coordinates as $X^{\mu}(\sigma, \tau)=X_{c l}^{\mu}+\xi^{\mu} ; X_{c l}^{\mu}$ being a classical solution of string coordinates and $\xi^{\mu}$ being the fluctuation. One can compute the $\beta$-function perturbatively as has been the practice and set it to zero in order to derive constraints on the background. A covariant formulations is to adopt Riemann normal coordinate expansion method. The point made by Das and Sathiapalan is described in sequel. When one carries out loop expansion for the $\sigma$-model it is renormalizable at each order in perturbation theory. However, for some choice of target space, when the loop expansion is summed to all orders there are new divergences. These divergences cannot be eliminated from the terms present in the starting Lagrangian. In order to remove this divergence, it is essential to introduce higher dimensional operators. In other words, for the case at hand, the graviton vertex is $\gamma^{a b} h_{\mu \nu} \partial_{a} \xi^{\mu} \partial_{b} \xi^{\nu}$. The new term has four terms like $\partial \xi \partial \xi \partial \xi \partial \xi$ and contracted with a fourth rank tensor (we shall discuss its precise form later). However, conformal invariance imposes additional constraints on the structure of the new piece we incorporate i.e. the new vertex operator that is required to eliminate the fresh divergence. The resulting constraint turns out to be precisely the equations of motion for the first excited massive level of the closed string. Indeed, it describes, in this case a three point function for graviton-graviton-M, M being (symbolically) the first excited massive state. Thus the presence of nonremormalizable operator of this type has important consequences. The consistency of the theory will demand, if we include such a term, more and more excited levels to be included in the effective action and eventually the entire tower of stringy states be added. Of course, string field theory is the proper arena to address and investigate these issues. Nevertheless, it is obvious that for the sake of consistency of string theory, in the first quantized frame work, excited levels have an important role.

It has been conjectured that excited stringy states might possess local symmetries $[61,62,64,63,65,66,67,68]$. This idea has been pursued from time to time and there are evidences that such symmetries manifest themselves, even in the first quantized approach. A conjecture was first put forward in the Hamiltonian phase space approach where the local symmetries associated with graviton and antisymmetric tensor (in the massless sector) were unraveled through introduction of certain canonical transformations. In fact, the Ward identities revealed the manifestation of such symmetries [61]. Subsequently, several authors have carefully studied the proposal and have found evidences for higher symmetries explicitly for first few massive levels $[65,66, ?]$. Moreover, there have been proposals to explore possible existence of stringy states at the accessible energy scales [69, 70, 71, 72].

The role of excited massive states have come to light in the study of Planckian energy scattering of string states. For example when Planckian energy scattering of gravitons are considered, it is essential that effects of all string states are properly accounted for in order to get some of the desired features of the scattering amplitude $[73,75,76,77,78]$. Moreover, Gross [79] has conjectured that in ultra high energy scatterings, when masses of string states play no significant role, there might be a 
hidden infinite dimensional symmetry in string theory. Furthermore, scattering of all stringy state amplitudes will be related to a single amplitude. Finally, we mention that Vasiliev's theory of higher spin states has attracted a lot of attentions in recent years [80]. Although the entire programme, with inclusion of interactions, is yet to be completed, progress has been made to understand interactions in higher spin field theory from the string theoretic perspective.

In this optics, it is worth while to investigate duality symmetry associated with excited massive levels of closed string where $d$ of its spacial coordinates are compactified on $T^{d}$. We recall some salient results of T-duality in the frame work of the worldsheet theory and we focus on toroidal compactification for massless states in the worldsheet approach.

\subsection{Review of Properties Excited States}

Let us very quickly recapitulate some of the results of the previous section. We shall need these ingredients in what follows. $Y^{\alpha}(\sigma, \tau), \alpha, \beta=1,2, . . d$ are toroidally compact coordinate on $T^{d}$. The noncompact coordinates are $X^{\mu}(\sigma, \tau), \mu, \nu=0,1,2 . . D-1$ with $D+d=\hat{D}$. The corresponding backgrounds after dimensional reduction[22], for the metric, are $g_{\mu \nu}(X), A_{\mu \alpha}^{(1)}(X)$ and $G_{\alpha \beta}(X)$. The the 2-form B-field gives $B_{\mu \nu}(X), B_{\mu \alpha}$ and $B_{\alpha \beta}(X)$ when dimensionally reduced. It is assumed that all the backgrounds depend only spacetime string coordinates $X^{\mu}$. The gauge fields $A_{\mu \alpha}^{(1)}$ are associated with the isometries and $B_{\mu \alpha}$ are another set of gauge fields coming from dimensional reductions of the 2-form. It was shown, in the previous section that after introducing a set of dual coordinates $\tilde{Y}^{\alpha}$ the combined worldsheet equations of motion (of $Y$ and $\tilde{Y}$ ) can be cast in a duality covariant form. Note that if one resorts to the Hamiltonian formulation for a slightly simplified version of above compactification [95], the resulting Hamiltonian is expressed in duality invariant form. Our strategy will be to utilize the results of Hamiltonian formulation and adopt a simple compactification [58] procedure for the higher levels and unveil the duality symmetry for these states. Let us consider toroidal compactification where we set $G_{\alpha \beta}=\delta_{\alpha \beta}$ and $B_{\alpha \beta}=0$; in other words the radii of $T^{d}$ are set to unity as before. The stress energy momentum tensors used to compute the conformal weights are

$$
T_{++}=\frac{1}{2}\left(g_{\mu \nu}^{(0)} \partial X^{\mu} \partial X^{\nu}+\delta_{\alpha \beta} \partial Y^{\alpha} \partial Y^{\beta}\right)
$$

and

$$
T_{--}=\frac{1}{2}\left(g_{\mu \nu}^{(0)} \bar{\partial} X^{\mu} \bar{\partial} X^{\nu}+\bar{\partial} Y^{\alpha} \bar{\partial} Y^{\beta}\right)
$$

where $g_{\mu \nu}^{(0)}=\operatorname{diag}(1,-1,-1 .$.$) is the flat D-dimensional metric, \partial X^{\mu}=\dot{X}^{\mu}+X^{\prime \mu}$, $\partial Y^{\alpha}=\dot{Y}^{\alpha}+Y^{\alpha}, \bar{\partial} X^{\mu}=\dot{X}^{\mu}-X^{\prime \mu}$ and $\bar{\partial} Y^{\alpha}=\dot{Y}^{\alpha}-Y^{\prime \alpha}$; dot and 'prime' stand for 
derivatives with respect to $\tau$ and $\sigma$ here and everywhere. We define vertex functions as follows. The vertex operator of a given level is a sum of several vertex functions. A vertex operator of a given mass level is required to satisfy $(1,1)$ condition with respect to $\left(T_{++}, T_{--}\right)$. Consequently, the vertex functions, in general, are not independent and might satisfy certain relations as we shall see later. In certain cases, some of them might be 'gauged away' when we count physical degrees of freedom. We list below the vertex functions $[65,66]$ corresponding to the first massive level for the uncompactified, $\hat{D}$-dimensional spacetime. A vertex operator is sum of many vertex functions as given below for the first excited level. As we go to higher and higher levels, the number of vertex functions increase.

$$
\begin{gathered}
\hat{V}_{1}^{(1)}=A_{\hat{\mu} \hat{\nu}, \hat{\mu}^{\prime} \hat{\nu}^{\prime}}^{(1)}(X) \partial X^{\hat{\mu}} \partial X^{\hat{\nu}} \bar{\partial} X^{\hat{\mu}^{\prime}} \bar{\partial} X^{\hat{\nu}^{\prime}} \\
\hat{V}_{1}^{(2)}=A_{\hat{\mu} \hat{\nu}, \hat{\mu}^{\prime}}^{(2)}(X) \partial X^{\hat{\mu}} \partial X^{\hat{\nu}} \bar{\partial}^{2} X^{\hat{\mu}^{\prime}}, \quad \hat{V}_{1}^{(3)}=A_{\hat{\mu}, \hat{\mu}^{\prime} \hat{\nu}^{\prime}}^{(3)}(X) \partial^{2} X^{\hat{\mu}} \bar{\partial} X^{\hat{\mu}^{\prime}} \bar{\partial} X^{\hat{\nu}^{\prime}} \\
\hat{V}_{1}^{(4)}=A_{\hat{\mu}, \hat{\mu}^{\prime}}^{(4)}(X) \partial^{2} X^{\hat{\mu}} \bar{\partial}^{2} X^{\hat{\mu}^{\prime}}
\end{gathered}
$$

The subscript ' 1 ' appearing in $\hat{V}_{1}^{(1)}$ is indicative of the fact that these vertex functions correspond to ones for the first excited massive level. The vertex operator is

$$
\hat{\Phi}_{1}=\sum_{1}^{4} \hat{V}_{1}^{(i)}
$$

Notices that the tensor indices are labeled with unprimed and primed indices. This convention is adopted to keep track of the operators (or oscillators in mode expansions of $X^{\hat{\mu}}$ ) coming from the right moving sector such as $\partial X^{\hat{\mu}}$ and from the left moving sector, $\bar{\partial} X^{\hat{\mu}^{\prime}}$, or powers of $\partial, \bar{\partial}$ acting on $X^{\hat{\mu}}$. It facilitates our future computation and will be useful notation when we dwell on duality symmetry in sequel. It is a straight forward calculation to obtain the constraints on the vertex functions $V_{1}^{(i)}$ (actually conditions on the $X$-dependent tensors, $\left.A^{(i)}\right)$ if they are to be $(1,1)$ primaries with respective to $T_{ \pm \pm}$. We follow the methods of $[65,66]$ and summarize the relevant results below. These will be utilized when we explore the associated of T-duality properties of these vertex operators for the compactified scenario. Note that each of the vertex functions, $\left(V_{1}^{(2)}-V_{1}^{(4)}\right)$, is not $(1,1)$ on its own; however, $V^{(1)}$ is $(1,1)$ as is easily verified. Second point, we mention in passing, is that conformal invariance imposes two types of constraints on these vertex functions: each one satisfies a massshell condition (recall that same is true for tachyon and all massless vertex operators) and gauge (or transversality) conditions which is also known for all the massless sectors. These are listed below

$$
\left(\hat{\nabla}^{2}-2\right) A_{\hat{\mu} \hat{\nu}, \hat{\mu}^{\prime} \hat{\nu}^{\prime}}^{(1)}(X)=0, \quad\left(\hat{\nabla}^{2}-2\right) A_{\hat{\mu} \hat{\nu}, \hat{\mu}^{\prime}}^{(2)}(X)=0,
$$


and

$$
\left(\hat{\nabla}^{2}-2\right) A_{\hat{\mu}, \hat{\mu}^{\prime} \hat{\nu}^{\prime}}^{(3)}(X)=0, \quad\left(\hat{\nabla}^{2}-2\right) A_{\hat{\mu}, \hat{\mu}^{\prime}}^{(4)}(X)=0
$$

The $\hat{D}$-dimensional Laplacian, $\hat{\nabla}^{2}$, is defined in term of the flat spacetime metric. The mass levels are in in units of the string scale which has been set to one in eqs.(88) and (89). The four vertex functions also are related through following equations

$$
A_{\hat{\mu} \hat{\nu}, \hat{\mu}^{\prime}}^{(2)}=\partial^{\hat{\nu}^{\prime}} A_{\hat{\mu} \hat{\nu}, \hat{\mu}^{\prime} \hat{\nu}^{\prime}}^{(1)}, \quad A_{\hat{\mu}, \hat{\mu}^{\prime} \hat{\nu}^{\prime}}^{(3)}=\partial^{\hat{\nu}} A_{\hat{\mu} \hat{\nu}, \hat{\mu}^{\prime} \hat{\nu}^{\prime}}^{(1)}, \quad A_{\hat{\mu}, \hat{\mu}^{\prime}}^{(4)}=\partial^{\hat{\nu}^{\prime}} \partial^{\hat{\nu}} A_{\hat{\mu} \hat{\nu}, \hat{\mu}^{\prime} \hat{\nu}^{\prime}}^{(1)}
$$

Here $\partial^{\hat{\mu}}$ etc. stand for partial derivatives with respect to spacetime coordinates. Furthermore, besides eqs. (88),(89) and eq. (90) there are further constraints, like gauge conditions, which also follow from the requirements of that the vertex functions be $(1,1)$ primaries $[65,66]$

$$
A_{\hat{\mu}}^{(1) \hat{\mu}} \hat{\mu}^{\prime} \hat{\nu}^{\prime}+2 \partial^{\hat{\mu}} \partial^{\hat{\nu}} A_{\hat{\mu} \hat{\nu}, \hat{\mu}^{\prime} \hat{\nu}^{\prime}}^{(1)}=0, \quad \text { and } \quad A_{\hat{\mu} \hat{\nu}, \hat{\mu}^{\prime}}^{(1)} \hat{\mu}^{\prime}+2 \partial^{\hat{\mu}^{\prime}} \partial^{\hat{\nu}^{\prime}} A_{\hat{\mu} \hat{\nu}, \hat{\mu}^{\prime} \hat{\nu}^{\prime}}^{(1)}=0
$$

The above relations, eq.(90) and eq.(91), will be useful for our investigation of the duality in what follows.

Let us very briefly recapitulate how the T-duality group $O(d, d)$ plays an important role in the worldsheet Hamiltonian description of a closed string compactified on $T^{d}$. We shall proceed in two steps.

However, our attention will be on the weak field approximation and therefore, we shall briefly discuss the $O(d, d)$ invariance of graviton vertex operator (along compact directions), $h_{\alpha \beta^{\prime}} \partial Y^{\alpha} \bar{\partial} Y^{\beta^{\prime}}$; the argument can be extended for the case of weak $B_{\alpha \beta}$ in an analogous manner. Note that, in this approximation, the conjugate momentum $P_{\alpha}=\delta_{\alpha \beta} \dot{Y}^{\alpha}$ and all the indices are raised and lowered by $\delta^{\alpha \beta}$ and $\delta_{\alpha \beta}$ respectively. Thus the vertex operator takes the form

$$
V_{h}=h^{\alpha \beta^{\prime}} P_{\alpha} P_{\beta}-h_{\alpha \beta^{\prime}} Y^{\prime \alpha} Y^{\prime \beta^{\prime}}-h_{\beta^{\prime}}^{\alpha} P_{\alpha} Y^{\prime \beta^{\prime}}+h_{\beta}^{\alpha^{\prime}} P_{\alpha^{\prime}} Y^{\prime \beta}
$$

We adopt Hassan-Sen compactification scheme where the metric assumes a block diagonal form (i.e. the gauge fields associated with the isometries are set to zero)

$$
g_{\hat{\mu} \nu}=\left(\begin{array}{cc}
g_{\mu \nu} & 0 \\
0 & G_{\alpha \beta}
\end{array}\right)
$$

and correspondingly define the $O(d, d)$ vector

$$
\mathcal{W}=\left(\begin{array}{c}
P_{\alpha} \\
Y^{\prime \alpha}
\end{array}\right)
$$

Note that the four terms in (92) can combines to express in an $O(d, d)$ variant form, once we recognize that the first two terms can be written in terms of the $O(d, d)$ vector $\mathcal{W}$ the product $P Y^{\prime}$ needs some careful handling; we are not canceling out the 
last two terms in (92) since we continue to maintain distinctions between primed and unprimed indices. We might express $P_{\alpha}$ and $Y^{\prime \beta}$ as projected $O(d, d)$ vectors of $\mathcal{W}$ contracted with a suitable a suitable tensor and rewrite (92) in the following form

$$
V_{h}=H_{m n} \mathcal{W}^{m} \mathcal{W}^{n}-K_{m}^{n} \mathcal{W}^{m} \mathcal{W}_{n}
$$

Thus $V_{h}$, above will be $O(d, d)$ invariant $H$ and $K$ if satisfy following transformation properties along with the vectors $\left\{\mathcal{W}_{m}\right\}$

$$
H_{m n} \rightarrow \Omega_{n}^{m^{\prime}} \Omega_{n}^{n^{\prime}} H_{m^{\prime} n^{\prime}}, \mathcal{W}^{m} \rightarrow \Omega_{m^{\prime}}^{m} \mathcal{W}^{m^{\prime}}, K_{m}^{n} \rightarrow \Omega_{m}^{m^{\prime}} \Omega_{n^{\prime}}^{n} K_{m^{\prime}}^{n^{\prime}}
$$

A comment is in order here. We know that $h_{\alpha \beta}$, in $d$-dimensions has $\frac{d(d+1)}{2}$ components (when we do not impose tracelessness condition on $h$ ). However, counting the number of components of $H_{m n}$ shows that they exceed those of $h_{\alpha \beta}$. This is not surprising. When we expressed the Hamiltonian in T-duality invariant form, we introduced $M$-matrix which belongs to $O(d, d)$. It has $d^{2}-d$ components whereas $G+B$ have only $d^{2}$ components. Thus expressing the canonical Hamiltonian in T-duality invariant form has cost us these extra components. However, the physical degrees of freedom are the same. A careful analysis [22] shows that the T-duality group is $\frac{O(d, d)}{O(d) \otimes O(d)}$. On this occasion, we may argue that we had to pay a price to construct the duality invariant vertex operator. This argument holds when we construct duality invariant vertex functions for excited levels. Note that the inner product of an $O(d, d)$ vector, $T_{m}$ with $\mathcal{W}^{m}, T_{m} \mathcal{W}^{m}$ is to be interpreted as follows: $T_{m} \mathcal{W}^{m}=T^{\alpha} P_{\alpha}+T_{\alpha} Y^{\alpha}$. Moreover, all $O(d, d)$ tensor indices, $k, l, m, n, \ldots$, are raised and lowered by the the metric $\eta$, whereas the indices of $P_{\alpha}$ and $Y^{\prime \alpha}$ are raised and lowered by $\delta^{\alpha \beta}$ and $\delta_{\alpha \beta}$ respectively.

\subsection{T-duality Symmetry of Vertex Operators of Excited States}

Let us examine T-duality properties of the first excited massive level where we adopt a simple compactification scheme. We focus the attention on $V_{1}^{(1)}$ as an example. Note that if we follow the toroidal compactification scheme adopted in [22] in the context of worldsheet duality, for the case at hand, the vertex function $A_{\hat{\mu} \hat{\nu}, \hat{\mu}^{\prime} \hat{\nu}^{\prime}}^{(1)}(X) \partial X^{\hat{\mu}} \partial X^{\hat{\nu}} \bar{\partial} X^{\hat{\mu}^{\prime}} \bar{\partial} X^{\hat{\nu}^{\prime}}$ will decompose into following forms: (i) A tensor $A_{\mu \nu, \mu^{\prime} \nu^{\prime}}^{(1)}$, one which has all Lorentz indices (ii) another which has three Lorentz indices and one index corresponding to compact directions, (iii) a tensor with two Lorentz indices and two indices in compact directions, (iv) another, which has a single Lorentz index and three indices in in internal directions and (v) a tensor with all indices corresponding to compact directions i.e. $A_{\alpha \beta, \alpha^{\prime} \beta^{\prime}}^{(1)}$. It is obvious these tensors with be suitably contracted with $\partial X^{\mu}, \bar{\partial} X^{\mu}, \partial Y^{\alpha}, \bar{\partial} Y^{\alpha}$ with all allowed combinations. We adopt, to start with, a compactification scheme where only $A_{\alpha \beta, \alpha^{\prime} \beta^{\prime}}^{(1)}$ is present and the tensors with mixed indices are absent. We shall return to more general case later. We 
may allow the presence of $A_{\mu \nu, \mu^{\prime} \nu^{\prime}}^{(1)}$; note however, that its presence is not very essential for the discuss of T-duality symmetry since the spacetime tensors and coordinates are assumed to be inert under the T-duality transformations, as a consequence this term will be duality invariant on its own right. This is the line of argument advanced by us recently [23]. Therefore, we shall deal with a single vertex function to discuss T-duality symmetry as a prelude

$$
V_{1}^{(1)}=A_{\alpha \beta, \alpha^{\prime} \beta^{\prime}}^{(1)}(X) \partial Y^{\alpha} \partial Y^{\beta} \bar{\partial} Y^{\alpha^{\prime}} \bar{\partial} Y^{\beta^{\prime}}
$$

As argued earlier, if we expand the expression for $V_{1}^{(1)}$, eq.(97), out in terms of $P_{\alpha}$ and $Y^{\prime \alpha}$ we get terms of the following type contacted with the tensor $A_{\alpha \beta, \alpha^{\prime} \beta^{\prime}}^{(1)}(X)$; note that we do not use any symmetry(antisymmetry) properties of this tensor under $\alpha \leftrightarrow \beta$ and $\alpha^{\prime} \leftrightarrow \beta^{\prime}$. Although we express the vertex function in terms of $Y^{\prime}$ and $P$, we still like to retain the memory whether these terms came from left movers or right movers. The full expression for the vertex function is classified into five types. These are listed below:

(I) All are $P^{\alpha}$ s (index raised by $\left.\delta^{\alpha \beta}\right)$ :

$A_{\alpha \beta, \alpha^{\prime} \beta^{\prime}}^{(1)}(X) P^{\alpha} P^{\beta} P^{\alpha^{\prime}} P^{\beta^{\prime}}$.

(II) All are $Y^{\prime \alpha}$ 's:

$A_{\alpha \beta, \alpha^{\prime} \beta^{\prime}}^{(1)}(X) Y^{\prime \alpha} Y^{\prime \beta} Y^{\prime \alpha^{\prime}} Y^{\prime \beta^{\prime}}$.

(III) The four terms with three $P^{\alpha}$ 's are:

- $A_{\alpha \beta, \alpha^{\prime} \beta^{\prime}}^{(1)}(X) P^{\alpha} P^{\beta} P^{\alpha^{\prime}} Y^{\prime \beta^{\prime}}, \quad-A_{\alpha \beta, \alpha^{\prime} \beta^{\prime}}^{(1)}(X) P^{\alpha} P^{\beta} Y^{\prime \alpha^{\prime}} P^{\beta^{\prime}}$,

$A_{\alpha \beta, \alpha^{\prime} \beta^{\prime}}^{(1)}(X) P^{\alpha} Y^{\prime \beta} P^{\alpha^{\prime}} P^{\beta^{\prime}}, \quad A_{\alpha \beta, \alpha^{\prime} \beta^{\prime}}^{(1)}(X) Y^{\prime \alpha} P^{\beta} P^{\alpha^{\prime}} P^{\beta^{\prime}}$

(IV) The four terms with three $Y^{\prime \alpha}$ s which will eventually combine with the terms in (III) when we study T-duality property:

$-A_{\alpha \beta, \alpha^{\prime} \beta^{\prime}}^{(1)}(X) Y^{\prime \alpha} Y^{\prime \beta} P^{\alpha^{\prime}} Y^{\prime \beta^{\prime}}, \quad-A_{\alpha \beta, \alpha^{\prime} \beta^{\prime}}^{(1)}(X) Y^{\prime \alpha} Y^{\prime \beta} Y^{\prime \alpha^{\prime}} P^{\beta^{\prime}}$, $A_{\alpha \beta, \alpha^{\prime} \beta^{\prime}}^{(1)}(X) P^{\alpha} Y^{\prime \beta} Y^{\prime \alpha^{\prime}} Y^{\prime \beta^{\prime}}, \quad A_{\alpha \beta, \alpha^{\prime} \beta^{\prime}}^{(1)}(X) Y^{\prime \alpha} P^{\beta} Y^{\prime \alpha^{\prime}} Y^{\prime \beta^{\prime}}(\mathrm{V})$ There are six terms, each of which is a product of a pair of momenta $\left(P^{\alpha}\right.$ and a pair $Y^{\alpha}$ :

$A_{\alpha \beta, \alpha \beta^{\prime}}^{(1)} P^{\alpha} P^{\beta} Y^{\prime \alpha^{\prime}} Y^{\prime \beta^{\prime}}, \quad A_{\alpha \beta, \alpha \beta^{\prime}}^{(1)} Y^{\prime \alpha} Y^{\prime \beta} P^{\alpha^{\prime}} P^{\beta^{\prime}}$,

$-A_{\alpha \beta, \alpha \beta^{\prime}}^{(1)} P^{\alpha} Y^{\prime \beta} P^{\alpha^{\prime}} Y^{\prime \beta^{\prime}}, \quad-A_{\alpha \beta, \alpha \beta^{\prime}}^{(1)} Y^{\prime \alpha} P^{\beta} Y^{\prime \alpha^{\prime}} P^{\beta^{\prime}}$,

$-A_{\alpha \beta, \alpha \beta^{\prime}}^{(1)} Y^{\prime \alpha} P^{\beta} Y^{\prime \alpha^{\prime}} P^{\beta^{\prime}}, \quad-A_{\alpha \beta, \alpha \beta^{\prime}}^{(1)} Y^{\prime \alpha} P^{\beta} P^{\alpha^{\prime}} Y^{\prime \beta^{\prime}}$

A careful inspection of the above terms leads us to conclude that class (I) and class (II) have the right structures to form an $O(d, d)$ invariant term when we identify combinations of $P, Y^{\prime}$ to compose the $O(d, d)$ vector $\mathcal{W}$. Similarly, class (III) (with the product of thee $P$ and one $Y^{\prime}$ ) will combine with the class (IV) which has opposite number of momenta and $Y^{\prime \prime}$ s. These two (classes) combine to give us another $O(d, d)$ invariant piece. Note that $\left(\begin{array}{c}P \\ Y^{\prime}\end{array}\right)$ can be flipped $\left(P^{\alpha}\right.$ and $Y^{\prime \alpha}$ interchanged in the column) by operating the $\eta$-matrix on the $\mathcal{W}$ vector. It is just like flipping a down spin Pauli spinor to up spin state. Finally, the class $(\mathrm{V})$ is a product of a pair of $Y^{\prime}$ and a pair of $P$; therefore, this class can be cast in duality invariant form. In order 
to observe it more transparently, let us consider the two terms together in class (I) and in class (II) we can construct two doublets

$$
\left(\begin{array}{ll}
A_{\alpha \beta, \alpha^{\prime} \beta^{\prime}}^{(1)} & A_{\alpha \beta, \alpha^{\prime} \beta^{\prime}}^{(1)}
\end{array}\right), \quad\left(\begin{array}{c}
P^{\alpha} P^{\beta} P^{\alpha^{\prime}} P^{\beta^{\prime}} \\
Y^{\prime \alpha} Y^{\prime \beta} Y^{\prime \alpha^{\prime}} Y^{\prime \beta^{\prime}}
\end{array}\right)
$$

If we take inner product it will be T-duality invariant. This procedure can be extended to class (III) and class (IV) pairs; moreover the terms in class (V) can also be cast in the requisite form. However, this prescription is not very efficient when we consider higher and higher massive levels where the vertex operators will have increasing number of terms. Therefore, we propose the following alternative.

We can introduce following types of vertex operators in terms of the $O(d, d)$ vectors $\mathcal{W}^{n}$ :

$B_{k l, m^{\prime} n^{\prime}}^{(1)}(X) \mathcal{W}^{k} \mathcal{W} l^{l} \mathcal{W}^{m^{\prime}} \mathcal{W}^{n^{\prime}}, B_{k l, m^{\prime} n^{\prime}}^{(2)}(X) \mathcal{W}^{k}(\eta \mathcal{W})^{m^{\prime}} \mathcal{W}^{n^{\prime}}, B_{k l, m^{\prime} n^{\prime}}^{(3)}(X) \mathcal{W}^{k} \mathcal{W}^{l} \mathcal{W}^{m^{\prime}}(\eta \mathcal{W})^{n^{\prime}}(99)$

$\eta$ 's have been inserted to take into account flipping of $P$ and $Y^{\prime}$. Notice that the new vertex operators will be $O(d, d)$ invariant if the tensors $B^{(1)}, B^{(2)}$ and $B^{(3)}$ transform as follows under $O(d, d)$

$$
B^{(i)} k l, m^{\prime} n^{\prime} \rightarrow \Omega_{k}^{p} \Omega_{l}^{q} \Omega_{m^{\prime}}^{p^{\prime}} \Omega_{n^{\prime}}^{q^{\prime}} B_{p q, p^{\prime} q^{\prime}}^{(i)}, \quad \Omega \in O(d, d)
$$

since $\mathcal{W}^{k} \rightarrow \Omega_{l}^{k} \mathcal{W}^{l}$. We draw attention of the reader to the following points: (i) The prime and unprimed indices have been maintained even at this stage to keep track of the fact that certain momenta and $Y^{\prime}$ originate from the left moving sector and some other pairs from right moving sector. (ii) The $O(d, d)$ metric $\eta$ is used to raise and lower indices of the corresponding vectors and tensors. (iii) There are three tensors $B^{(i)}$, their linear combinations are related to $A^{(1)}$ once one compares all the terms in (99) with the terms collected in class (I) - class (V). (iv) Note also that we have not included a term which has two $\eta$ 's; one introduced between a pair of $\mathcal{W}$ coming from right movers and another between left movers. Such term amounts to 'double flip' and essentially will be equivalent to the term $B^{(1)}$ since the two $\eta$ 's can raise all the indices of the first vertex.

Let us consider the following three vertex functions in the present compactification scheme

$$
V_{1}^{(2)}=A_{\alpha \beta, \alpha^{\prime}}^{(2)}(X) \partial Y^{\alpha} \partial Y^{\beta} \bar{\partial}^{2} Y^{\alpha^{\prime}}, \quad V_{1}^{(3)}=A_{\alpha, \alpha^{\prime} \beta^{\prime}}^{(3)}(X) \partial^{2} Y^{\alpha} \partial Y^{\alpha^{\prime}} \partial Y^{\beta^{\prime}}
$$

and

$$
V_{1}^{(4)}=A_{\alpha, \alpha^{\prime}}^{(4)}(X) \partial^{2} Y^{\alpha} \bar{\partial}^{2} Y^{\alpha^{\prime}}
$$

If we demand that these vertex functions be $(1,1)$ primaries then, it follows from $(90)$ that these vertex operators should vanish, since all the tensor indices correspond to compact directions whereas the vertex functions depend only on $X^{\mu}$. Thus partial 
derivatives, $\partial^{\alpha}$ acting on $A^{(1)}$ vanishes; thus $A^{(2)}, A^{(3)}$ and $A^{(4)}$, consequently, all vanish since they are related to derivatives of $A^{(1)}$ from the constraints alluded to earlier (90). However, one issue is to be borne in mind, in the context of the T-duality characteristics of these vertex functions, is that one does not impose constraints of conformal invariance to start with. Note that $V_{1}^{(2)}$ and $V_{1}^{(3)}$ have double derivative on one sector and two single derivatives on another sector of the world sheet coordinates from dimensional considerations. Thus we get $\tau$ and/or $\sigma$ derivatives of $P^{\alpha}, Y^{\prime \alpha^{\prime}}$ etc. as expected. Consequently, it becomes quite difficult to cast vertex functions in a Tduality invariant form when we have such class of vertex functions $\left(A^{(4)}\right.$ has similar property now with two double derivatives on each sector) if we follow the procedure adopted for $V^{(1)}$. Moreover, we shall continue to encounter these difficulties as we go to higher and higher excited states. One of the illustrative examples is to envisage a vertex function for the second massive level which has only $\partial$ and $\bar{\partial}$ acting of $Y$ 's maintaining the required dimensionality (we are dealing with compactified case here)

$$
V_{2}^{(1)}=C_{\alpha \beta \gamma, \alpha^{\prime} \beta^{\prime} \gamma^{\prime}}^{(1)} \partial Y^{\alpha} \partial Y^{\beta} \partial Y^{\gamma} \bar{\partial} Y^{\alpha^{\prime}} \bar{\partial} Y^{\beta^{\prime}} \bar{\partial} Y^{\gamma^{\prime}}
$$

We can carry out an analysis similar to the vertex function, $V_{1}^{(1)}$, of the first massive level and classify various pieces which can be combined to construct $O(d, d)$ invariant terms. Indeed, this check, although tedious, has been done to obtain T-duality invariant combinations. However, for the 2 nd massive level there are vertex functions of the form, $\partial^{2} Y^{\alpha} \partial Y^{\beta} \bar{\partial}^{3} Y^{\alpha^{\prime}}$, which are contracted with suitable tensor and there are many more terms. It is not easy to exhibit T-duality properties when there are higher order derivatives of $\sigma$ and $\tau$ acting on $O(d, d)$ vector composed of $P_{\alpha}$ and $Y^{\prime \alpha}$.

\subsection{T-duality of Vertex Operators of Arbitrary Massive State}

We propose a procedure to systematically organize various vertex operators which we describe in what follows. (i) The first observation is that the basic building blocks of vertex functions are $\partial Y^{\alpha}=P^{\alpha}+Y^{\alpha}$ and $\bar{\partial} Y^{\alpha^{\prime}}=P^{\alpha^{\prime}}-Y^{\prime \alpha^{\prime}}$. (ii) Each vertex function at a given level is either string of products of these basic blocks or these blocks are operated by $\partial$ and $\bar{\partial}$ respectively so that each vertex function at a given mass level has the desired dimensions. Note that it is not convenient to deal with $P^{\alpha}$ and $Y^{\alpha}$ separately in order to study the T-duality properties (in our approach) and the same is true for the combination $P \pm Y^{\prime}$. However, $P^{\alpha}$ and $Y^{\prime \alpha}$ can be projected out from the $O(d, d)$ vector, $\mathcal{W}$. Similarly, $\partial$ or $\bar{\partial}$ acting on $P \pm Y^{\prime}$ mixed mixed derivatives, in $\sigma$ and $\tau$, which is not convenient to deal with although these partial derivatives create objects of same conformal dimensions. Let us first introduce following projection operators for later conveniences [23]

$$
\mathbf{P}_{ \pm}=\frac{1}{2}\left(\mathbf{1} \pm \tilde{\sigma_{3}}\right)
$$


where $\mathbf{1}$ is $2 d \times 2 d$ unit matrix and $\tilde{\sigma_{3}}=\left(\begin{array}{cc}\mathbf{1} & 0 \\ 0 & -\mathbf{1}\end{array}\right)$ and the diagonal entries $(\mathbf{1},-\mathbf{1})$ stand for $d \times d$ unit matrices. It is easy to check that the projection operators are $O(d, d)$ matrices since each one of them is. We project out two $O(d, d)$ vectors as follows

$$
P=\mathbf{P}_{+} \mathcal{W}, \quad Y^{\prime}=\mathbf{P}_{-} \mathcal{W}
$$

Therefore,

$$
P+Y^{\prime}=\frac{1}{2}\left(\mathbf{P}_{+} \mathcal{W}+\eta \mathbf{P}_{-} \mathcal{W}\right), \quad P-Y^{\prime}=\frac{1}{2}\left(\mathbf{P}_{+} \mathcal{W}-\eta \mathbf{P}_{-} \mathcal{W}\right)
$$

notice that $\eta$ flips lower component $Y^{\prime}$ vector to an upper component one. Thus when we have only products of $P+Y^{\prime}$ and $P-Y^{\prime}$, we can express them first as products of $O(d, d)$ vector and subsequently contract their indices with appropriate tensors endowed with $O(d, d)$ indices. Next we deal with worldsheet partial derivatives $\partial$ and $\bar{\partial}$ operating on basic building blocks. Let us define [23]

$$
\Delta_{\tau}=\mathbf{P}_{+} \partial_{\tau}, \quad \Delta_{\sigma}=\mathbf{P}_{+} \partial_{\sigma} \quad \text { and } \quad \Delta_{ \pm}(\tau, \sigma)=\frac{1}{2}\left(\Delta_{\tau} \pm \Delta_{\sigma}\right)
$$

Therefore,

$$
\partial\left(P+Y^{\prime}\right)=\Delta_{+}(\tau, \sigma)\left(\mathbf{P}_{+} \mathcal{W}+\eta \mathbf{P}_{-} \mathcal{W}\right)
$$

Thus the above expression is an $O(d, d)$ vector. Similarly, when $\bar{\partial}$ operates on $P-Y^{\prime}$, we can express it as

$$
\bar{\partial}\left(P-Y^{\prime}\right)=\Delta_{-}(\tau, \sigma)\left(\mathbf{P}_{+} \mathcal{W}-\eta \mathbf{P}_{-} \mathcal{W}\right)
$$

The vertex functions we have considered in eqs.(97) and (103) which are expressed as only string of products of $\partial Y^{\alpha}$ and $\bar{\partial} Y^{\alpha^{\prime}}$ can be rewritten in terms of the $O(d, d)$ vectors $\mathcal{W}$ and subsequently contracted with suitable $O(d, d)$ tensors. We remind the reader that, now familiar, $M$-matrix which expresses the Hamiltonian in $O(d, d)$ invariant form is also parametrized in terms of backgrounds $G_{\alpha \beta}$ and $B_{\alpha \beta}$. Let us turn our attentions to the other three vertex functions appearing in (101) and (102). The procedure outlined above can be adopted to cast $V_{1}^{(2)}, V_{1}^{(3)}$ and $V_{1}^{(4)}$ in a straight forward manner using the relations (108) and (109).

In order to illustrate the variety of vertex functions that can arise as we envisage higher excited states we list a few vertex operators from second massive level [?].

$$
V_{2}^{(2)}=C_{\alpha \beta, \alpha^{\prime} \beta^{\prime} \gamma^{\prime}}^{(2)} \partial^{2} Y^{\alpha} \partial Y^{\beta} \bar{\partial} Y^{\alpha^{\prime}} \bar{\partial} Y^{\beta^{\prime}} \bar{\partial} Y^{\gamma^{\prime}}+C_{\alpha \beta \gamma, \alpha^{\prime} \beta^{\prime}}^{(3)} \partial Y^{\alpha} \partial Y^{\beta} \partial Y^{\gamma} \bar{\partial}^{2} Y^{\alpha^{\prime}} \bar{\partial} Y^{\beta^{\prime}}
$$


These vertex operators have a term of the form $\partial^{2} Y^{\alpha}$ or $\bar{\partial}^{2} Y^{\alpha^{\prime}}$ and rest of the structure is decided by dimensional considerations. The next class is the one which have either a $\partial^{3} Y$ or $\bar{\partial}^{3} Y$

$$
V_{2}^{(3)}=C_{\alpha, \alpha^{\prime} \beta^{\prime} \gamma^{\prime}}^{(4)} \partial^{3} Y^{\alpha} Y^{\alpha^{\prime}} \bar{\partial} Y^{\beta^{\prime}} \bar{\partial} Y^{\gamma^{\prime}}+C_{\alpha \beta \gamma, \alpha^{\prime}}^{(5)} \partial Y^{\alpha} \partial Y^{\beta} \partial Y^{\gamma} \bar{\partial}^{3} Y^{\alpha^{\prime}}
$$

Another type of term is

$$
V_{2}^{(4)}=C_{\alpha \beta, \alpha^{\prime} \beta^{\prime}}^{(6)} \partial^{2} Y^{\alpha} \partial Y^{\beta} \bar{\partial}^{2} Y^{\alpha^{\prime}} \bar{\partial} Y^{\beta^{\prime}}
$$

There are two terms

$$
V_{2}^{(5)}=C_{\alpha \alpha^{\prime} \beta^{\prime}}^{(7)} \partial^{3} Y^{\alpha} \bar{\partial}^{2} Y^{\alpha^{\prime}} \bar{\partial} Y^{\beta^{\prime}}+C_{\alpha \beta, \alpha^{\prime}}^{(8)} \partial^{2} Y^{\alpha} \partial Y^{\beta} \bar{\partial}^{3} Y^{\alpha^{\prime}}
$$

and the last term is

$$
V_{2}^{(6)}=C_{\alpha, \alpha^{\prime}}^{(9)} \partial^{3} Y^{\alpha} \bar{\partial}^{3} Y^{\alpha^{\prime}}
$$

The tensors $C^{(2)}-C^{(9)}$ appearing in eqs.(110-114) are all functions of $X^{\mu}$, independent of compact coordinates $Y^{\alpha}$, and constrained by requirements of conformal invariance (not necessarily nonvanishing in the compactification scheme we envisage). We observe from the structures of vertex operators $V_{2}^{(1)}-V_{2}^{(6)}$ that, each one with the combinations of the terms will be $O(d, d)$ invariant when we follow the prescriptions of introducing projection operators, rewrite the combinations $P+Y^{\prime}$ and $P-Y^{\prime}$ as $O(d, d)$ vectors and convert $\partial$ and $\bar{\partial}$ to $\Delta_{ \pm}(\tau, \sigma)$ to operate on $P \pm Y^{\prime}$ (re-expressed in terms of the projected $\mathcal{W}$ 's) respectively. Let us consider $n^{\text {th }}$ excited massive level as an example. The the dimension of all right movers obtained from products of $\partial Y$ higher powers of $\partial$ acting on $\partial Y$ should be $(n+1)$ and same hold good for the left moving sector as well. Consider the right moving sector of the type $\Pi_{1}^{n+1} \partial Y^{\alpha_{i}}$ and the left moving sector $\Pi_{1}^{n+1} \bar{\partial} Y^{\alpha_{i}^{\prime}}$ The vertex function is

$$
V_{\alpha_{1}, \alpha_{2} \ldots \alpha_{n+1}, \alpha_{1}^{\prime} \alpha_{2}^{\prime} \ldots \alpha_{n+1}^{\prime}}(X) \Pi_{1}^{n+1} \partial Y^{\alpha_{i}} \Pi_{1}^{n+1} \bar{\partial} Y^{\alpha_{i}^{\prime}}
$$

and these products of $\partial Y^{\alpha_{i}}$ and $\bar{\partial} Y^{\alpha_{i}^{\prime}}$ can be converted to products of $(n+1)$ projected $\mathcal{W}$ for right movers and $(n+1)$ projected $\mathcal{W}$ from left movers. Let us consider a for vertex function for a high level state. A generic vertex will have a structure

$\partial^{p} Y^{\alpha_{i}} \partial^{q} Y^{\alpha_{j}} \partial^{r} Y^{\alpha_{k}} . . \bar{\partial}^{p^{\prime}} Y^{\alpha_{i}^{\prime}} \bar{\partial}^{q^{\prime}} Y^{\alpha_{j}^{\prime}} \bar{\partial}^{r^{\prime}} Y^{\alpha_{k}^{\prime}} . ., p+q+r=n+1, p^{\prime}+q^{\prime}+r^{\prime}=n+(116)$

The product is an $O(d, d)$ tensor whose rank is decided by the constraints on sum

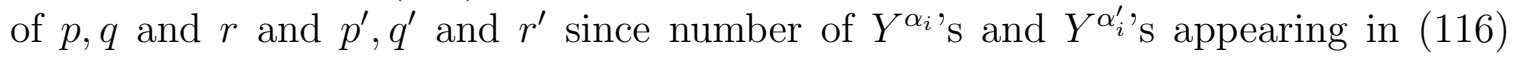
is determined from those conditions. Thus this tensor will be contracted with an appropriate tensor $T_{\alpha_{i} \alpha_{j} \alpha_{k} ., \alpha_{i}^{\prime} \alpha_{j}^{\prime} \alpha_{k} . .}(X)$ which will give us to a vertex function. Let us discuss how to express eq.(116) as a product of $O(d, d)$ vectors using the projection operators introduced earlier. 
(i) The first step is to rewrite

$\partial^{p} Y=\partial^{p-1}\left(P+Y^{\prime}\right), \quad \bar{\partial}^{p^{\prime}}\left(P-Y^{\prime}\right)=\bar{\partial}^{p^{\prime}-1}\left(P-Y^{\prime}\right)$

(ii) We arrive at

$\partial^{p-1}\left(P+Y^{\prime}\right)=\Delta_{+}{ }^{p-1}\left(P+Y^{\prime}\right), \quad \bar{\partial}^{p^{\prime}-1}\left(P-Y^{\prime}\right)=\Delta_{-}{ }^{p^{\prime}-1}\left(P-Y^{\prime}\right)$

from (108) and (109)

(iii) Finally, using the projection operators (106) we get

$\Delta_{+}{ }^{p-1}\left(P+Y^{\prime}\right)=\Delta_{+}{ }^{p-1}\left(\mathbf{P}_{+} \mathcal{W}+\eta \mathbf{P}_{-} \mathcal{W}\right), \quad \Delta^{p^{\prime}-1}\left(P-Y^{\prime}\right)=\Delta_{-}{ }^{p^{\prime}-1}\left(\left(\mathbf{P}_{+} \mathcal{W}-\eta \mathbf{P}_{-} \mathcal{W}\right)\right.$

Thus the products in (116) can be expressed as products of $O(d, d)$ vectors. We need to contract these indices with suitable $O(d, d)$ tensors which have the following form:

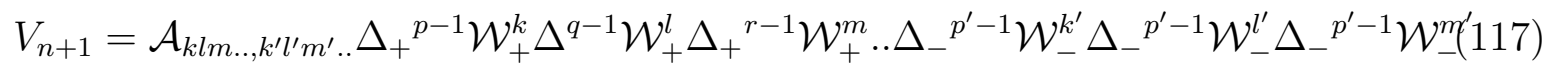

where $\mathcal{W}_{ \pm}=\left(\mathbf{P}_{+} \mathcal{W} \pm \eta \mathbf{P}_{-} \mathcal{W}\right)$ with $p+q+r=n+1$ and $p^{\prime}+q^{\prime}+r^{\prime}=n+1$. Note that superscripts $\left\{k, l, m ; k^{\prime}, l^{\prime}, m^{\prime}\right\}$ appearing on $\mathcal{W}_{ \pm}$in eq. (117) are the indices of the components of the projected $O(d, d)$ vectors. Moreover, $\mathcal{A}_{k l m . ., k^{\prime} l^{\prime} m^{\prime} . .}$ is $X$-dependent $O(d, d)$ tensor.

Now we turn our attention in another direction. So far we have dealt with those vertex functions which have only indices corresponding to compact directions. Therefore, all the massive excitations are scalars under $S O(D-1)$. Let us consider the first excited massive level to illustrate our strategy which can be generalized to any level. We claim that all vertex functions, for this level, are $O(d, d)$ invariant. We recall $X^{\mu}$ and tensors with only spacetime indices (i.e. $\mu, \nu, .$. etc.) a tensor transform trivially under the T-duality for these set of indices. Thus

$$
\tilde{V}_{1}^{(1)}=\tilde{A}_{\mu \nu, \mu^{\prime} \nu^{\prime}}^{(1)} \partial X^{\mu} \partial X^{\nu} \bar{\partial} X^{\mu^{\prime}} \bar{\partial} X^{\nu^{\prime}}
$$

is $O(d, d)$ invariant as per above prescription. Similarly, vertex functions:

$\tilde{A}_{\mu, \mu^{\prime} \nu^{\prime}}^{(2)} \partial^{2} X^{\mu} \bar{\partial} X^{\mu^{\prime}} \bar{\partial} X^{\nu^{\prime}}, \tilde{A}_{\mu \nu, \mu^{\prime}}^{(3)} \partial X^{\mu} \partial X^{\nu} \bar{\partial}^{2} X^{\mu^{\prime}}$ and $\tilde{A}_{\mu, \mu^{\prime}}^{(3)} \partial^{2} X^{\mu} \bar{\partial}^{2} X^{\mu^{\prime}}$ are also $O(d, d)$ invariant. Let us classify the vertex functions according to the spacetime and 'internal' indices they carry (with appropriate contractions of course).

(A) Vertex functions which have one Lorentz index and three internal indices: $\tilde{B}_{\mu \alpha, \alpha^{\prime} \beta^{\prime}}^{(1)} \partial X^{\mu} \partial Y^{\alpha} \bar{\partial} Y^{\alpha^{\prime}} \bar{\partial} Y^{\beta^{\prime}}+$ other terms by permuting the indices.

(B) Vertex functions which have two Lorentz indices and two internal indices: $\tilde{B}_{\mu \beta, \mu^{\prime} \beta^{\prime}}^{(2)} \partial X^{\mu} \partial Y^{\beta} \bar{\partial} X^{\mu^{\prime}} \bar{\partial} Y^{\beta^{\prime}}+$ other similar terms.

(C) Vertex functions with three Lorentz indices and one internal index:

$\tilde{B}_{\mu \nu, \mu^{\prime} \beta^{\prime}}^{(3)} \partial X^{\mu} \partial X^{\nu} \bar{\partial} X^{\mu^{\prime}} \bar{\partial}^{2} Y^{\beta^{\prime}}+$ other similar terms.

(D) Vertex functions of the type:

(i) $\tilde{B}_{\mu \nu, \alpha^{\prime}}^{(4)} \partial X^{\mu} \partial X^{\nu} \bar{\partial} Y^{\beta^{\prime}}+$ other similar terms.

(ii) $\tilde{B}_{\mu \beta, \alpha^{\prime}}^{(5)} \partial X^{\mu} \partial Y^{\alpha} \bar{\partial}^{2} Y^{\beta^{\prime}}+$ other similar terms.

(iii) Vertex functions with second derivatives:

$\tilde{B}_{\alpha, \mu^{\prime}}^{(6)} \partial^{2} Y^{\alpha} \bar{\partial}^{2} X^{\mu^{\prime}}$ and $\tilde{B}_{\mu, \alpha^{\prime}}^{(7)} \partial^{2} X^{\mu} \bar{\partial}^{2} Y^{\alpha^{\prime}}$

The vertex functions whose Lorentz index/indices are contracted with $\partial X^{\mu}, \partial^{2} X^{\mu}$, 
$\bar{\partial} X^{\mu^{\prime}}, \partial^{2} X^{\mu}$ will be inert under $O(d, d)$ rotations; however, rest of the indices correspond to internal indices and those are contracted with $\partial Y^{\alpha}, \bar{\partial} Y^{\alpha^{\prime}}, \partial^{2} Y^{\alpha}, \bar{\partial}^{2} Y^{\alpha^{\prime}}$ and so on. Moreover, the vertex functions considered above, (A)-(D), do not necessarily vanish unlike the cases when some vertex function, carrying only internal indices $\left(V_{1}^{(2)}-V_{1}^{(4)}\right)$, vanished as the consequences of conformal invariance i.e. that these are $(1,1)$ primaries. This conclusion can be easily verified from relations eqs. (90) and (91). We conclude that only the worldsheet variables with internal indices, such as $P \pm Y^{\prime}$ are relevant to construct $O(d, d)$ vectors which contract with corresponding indices of the relevant tensors. We have laid down a procedures to construct $O(d, d)$ vectors from $\partial Y^{\alpha}, \bar{\partial} Y^{\alpha^{\prime}}, \partial^{2} Y^{\alpha}, \bar{\partial}^{2} Y^{\alpha^{\prime}}$ and other higher derivative objects. For example, $\tilde{B}_{\mu \alpha, \alpha^{\prime} \beta^{\prime}}^{(1)} \partial X^{\mu} \partial Y^{\alpha} \bar{\partial} Y^{\alpha^{\prime}} \bar{\partial} Y^{\beta^{\prime}}$ has three 'internal' indices of $\tilde{B}^{(1)}$ contracted with $\partial Y^{\alpha} \bar{\partial} Y^{\alpha^{\prime}} \bar{\partial} Y^{\beta^{\prime}}$ and therefore, this vertex function will be converted to an $O(d, d)$ invariant vertex function which has a generic form

$$
\tilde{T}_{k, k^{\prime} l^{\prime}}^{(1)} \mathcal{W}^{k} \mathcal{W}^{k^{\prime}} \mathcal{W}^{l^{\prime}}
$$

This argument can be carried forward for all vertex functions at any massive level of the closed bosonic string. Moreover, the type of vertex functions discussed in (A)-(D) correspond to massive particles of various spins which fall into the representations of $S O(D-1)$. Therefore, we are also able to conclude that vertex functions for massive levels of a closed bosonic string can be cast in an $O(d, d)$ invariant form for every level following the procedure presented here.

\section{T-duality of NSR String}

We further explore the consequences of our proposal in the context of superstrings. First of all, let us discuss implications of $\sigma \leftrightarrow \tau$ T-duality for free superstring coordinates. Under this interchange, the two dimensional chiral bosonic coordinates transform as

$$
\partial_{ \pm} X_{L, R}^{\hat{\mu}}(\tau, \sigma) \rightarrow \pm \partial_{ \pm} X_{L, R}^{\hat{\mu}}(\tau, \sigma)
$$

since we can decompose $X^{\hat{\mu}}(\tau, \sigma)=X_{L}^{\hat{\mu}}(\tau+\sigma)+X_{R}^{\hat{\mu}}(\tau-\sigma)$ When we introduce their (worldsheet) superpartner chiral Majorana fermions, they transform as

$$
\psi_{L}^{\hat{\mu}}(\tau+\sigma) \rightarrow \psi_{L}^{\hat{\mu}}(\tau+\sigma), \quad \psi_{R}^{\hat{\mu}}(\tau-\sigma) \rightarrow-\psi_{R}^{\hat{\mu}}(\tau-\sigma)
$$

It is obvious that the canonical Hamiltonian associated with free theory will not be $\sigma \leftrightarrow \tau$ T-duality invariant since the fermionic part of the Lagrangian is first order (it is Dirac Lagrangian) and consequently the canonical conjugate of a fermion is fermion itself (modulo $\gamma$ matrix) unlike the bosonic coordinate where the conjugate momentum is the $\tau$-derivative. Therefore, revealing invariance of the Hamiltonian 
density under $O(d, d)$, T-duality, symmetry or alternatively deriving $O(d, d)$ covariant equations of motion face difficulties if we adopt an action expressed in terms of component fields. As we shall discuss later, it is most efficient to go over to the superfield formalism. In the past, there were certain obstacles to express the worldsheet evolution equations in $O(d, d)$ covariant form. We have overcome those difficulties recently [82] and equations of motion can be cast in T-duality covariant form, analogous to the bosonic case. We have adopted the NSR formalism and our discussions will be confined to the NS-NS sector all along.

A free superstring ( NSR string) action can be expressed as sum of actions for set of left moving and right moving bosons and fermions. Therefore, unlike the closed bosonic string case, we do not see the $P \leftrightarrow X^{\prime}$ duality (which is same as $\sigma \leftrightarrow \tau$ duality) so explicitly in the resulting Hamiltonian density in the presence of fermionic coordinates. In fact the more transparent duality symmetry is to study the transformation properties of left moving and right moving fields under $\sigma \leftrightarrow \tau$ interchange. The holomorphic fields do not change sign whereas antiholomorphic ones do. If we introduce constant backgrounds as in the case of closed bosonic string the analog of noncompact $O(\hat{D}, \hat{D})$ symmetry does not emerge so neatly. The target space duality for superstrings in the NSR formulation has been studied in the past, however, we feel that this problem deserves further attention.

Let us recapitulate evolution of NSR string in the background of massless excitations. One starts with the superworldsheet action in two dimensional superspace where the components of superfield are the bosonic coordinates, (NSR) Majorana fermions and auxiliary fields and the backgrounds are functions of the superfields. We expand the backgrounds in terms of component fields, eliminate the auxiliary fields in order to arrive at NSR superstring action in the presence of massless backgrounds with component the fermionic and boson fields only. If we envisage the case where backgrounds are independent of some of the coordinates (now backgrounds and their derivatives depend only on bosonic coordinates), then it is very hard to arrive at duality invariant/covariant equations as was achieved by Maharana and Schwarz [22]. Das and Maharana [83] considered NSR string action in superspace and adopted the technique introduced by Maharana and Schwarz [22] for the closed bosonic string to get analogous equations of motion. However, they were unable to arrive at at duality covariant equations of motion although they obtained interesting results for a special case. In this case the $Z_{2}$ duality conditions are recovered

$$
\partial_{ \pm} X_{L, R}^{\hat{\mu}} \rightarrow \pm \partial_{L, R} X^{\hat{\mu}}, \quad \psi_{L, R}^{\mu} \rightarrow \pm \psi_{L, R}^{\mu}
$$

Moreover, Siegel [84] considered superstring in superspace in a Hamiltonian phase space approach to study dualities. Subsequently, there have been attempts to reveal duality symmetries on superstring [85, 86, 87, 88]. Thus far worldsheet equations of motion for superstrings, transforming covariantly under duality transformation, have not been derived in a systematic manner at par with the results of closed bosonic string. 


\subsection{Superfield Formulation of NSR String and T-duality}

The NSR superstring action in two dimensional superspace is given by

$$
S=-\frac{1}{2} \int d \sigma d \tau d^{2} \theta \bar{D} \hat{\Phi}^{\hat{\mu}}\left(G_{\hat{\mu} \hat{\nu}}(\hat{\Phi})-\gamma_{5} B_{\hat{\mu} \hat{\nu}}(\hat{\Phi})\right) D \hat{\Phi}^{\hat{\nu}}
$$

We have adopted superorthonormal gauge in arriving at this action. Here $\hat{G}_{\hat{\mu \nu}}(\hat{\Phi})$ and $\hat{B}_{\hat{\mu \nu}}(\hat{\Phi})$ are the graviton and and 2-form backgrounds which depend on the superfield $\hat{\Phi}$. It has expansion in component fields as

$$
\hat{\Phi}^{\hat{\mu}}=X^{\hat{\mu}}+\bar{\theta} \psi^{\hat{\mu}}+\bar{\psi}^{\hat{\mu}} \theta+\frac{1}{2} \bar{\theta} \theta F^{\hat{\mu}}
$$

where $X^{\hat{\mu}}, \psi^{\hat{\mu}}$ and $F^{\hat{\mu}}$ are the bosonic, fermionic and auxiliary fields respectively. The covariant derivatives in superspace are defined to be

$$
D_{\alpha}=\frac{\partial}{\partial \bar{\theta}_{\alpha}}-i\left(\gamma^{a} \theta\right)_{\alpha} \partial_{a}, \quad \bar{D}_{\alpha}=-\frac{\partial}{\partial \theta_{\alpha}}+i\left(\bar{\theta} \gamma^{a}\right)_{\alpha} \partial_{a}
$$

where $\partial_{a}$ stands for worldsheet derivatives $(\sigma$ and $\tau$ ) and the convention for $\gamma$ matrices are

$$
\gamma^{0}=\left(\begin{array}{ll}
0 & 1 \\
1 & 0
\end{array}\right), \gamma^{1}=\left(\begin{array}{cc}
0 & -1 \\
1 & 0
\end{array}\right), \gamma_{5}=\gamma^{0} \gamma^{1}=\left(\begin{array}{cc}
1 & 0 \\
0 & -1
\end{array}\right)
$$

The resulting equations of motion from (123) are

$$
\bar{D}\left(G_{\hat{\mu} \hat{\nu}}(\hat{\Phi})-\gamma_{5} B_{\hat{\mu} \hat{\nu}}(\hat{\Phi})\right) D \hat{\Phi}^{\hat{\nu}}=0
$$

The equations for component fields can be obtained by expanding the backgrounds in terms of them and utilizing the definitions of superspace derivatives (125). Let us consider a compactification [58] scheme such that target space is compactified on $T^{d}$ : $\hat{\mathcal{M}}_{\hat{D}}=M_{D} \otimes T^{d}$. The metric and 2-form backgrounds are decomposed as

$$
G_{\hat{\mu} \hat{\nu}}=\left(\begin{array}{cc}
g_{\mu \nu}(\phi) & 0 \\
0 & G_{i j}(\phi)
\end{array}\right), B_{\hat{\mu} \hat{\nu}}=\left(\begin{array}{cc}
B_{\mu \nu}(\phi) & 0 \\
0 & B_{i j}(\phi)
\end{array}\right)
$$

Note that the backgrounds only depend on spacetime superfields, $\phi^{\mu}$. We decompose the superfields as

$$
\hat{\Phi}^{\hat{\mu}}=\left(\phi^{\mu}, W^{i}\right)
$$

where $\mu, \nu=0,1,2 . . D-1$ and $i, j=1,2, . . d$ with $\hat{D}=D+d$. Note that the two superfields have the expansions

$$
\phi^{\mu}=X^{\mu}+\bar{\theta} \psi^{\mu}+\bar{\psi}^{\mu} \theta+\frac{1}{2} \bar{\theta} \theta F^{\mu}
$$


and

$$
W^{i}=Y^{i}+\bar{\theta} \chi^{i}+\bar{\chi}^{i} \theta+\frac{1}{2} \bar{\theta} \theta F^{i}
$$

$\chi^{i}$ are two dimensional Majorana spinors. In this compactification scheme, the equations of motion for the superfields $\phi^{\mu}$ is exactly analogous to (127) where we replace $\hat{\Phi}$ with $\phi$ and the backgrounds with $g_{\mu \nu}(\phi)$ and $B_{\mu \nu}(\phi)$.

Let us focus attention on the evolution equations and the dynamics of superfields along compact directions [82]. The action is

$$
S=-\frac{1}{2} \int d \sigma d \tau d^{2} \theta \bar{D} W^{i}\left(G_{i j}(\phi)-\gamma_{5} B_{i j}(\phi)\right) D W^{j}
$$

The superderivatives are defines in (125) above. The equations of motion for $\left\{W^{i}\right\}$ are

$$
\bar{D}\left(\left[G_{i j}(\phi)-\gamma_{5} B_{i j}(\phi)\right] D W^{j}\right)=0
$$

In view of the fact that $G$ and $B$ depend only on $\phi^{\mu}$, we may introduce a dual free superfield $\widetilde{W}_{i}$ satisfying following equation locally which is consistent with (133)

$$
\left(G_{i j}(\phi)-\gamma_{5} B_{i j}(\phi)\right) D W^{j}=D \widetilde{W}_{i}
$$

and the dual superfield satisfies the constraint: $\bar{D} D \widetilde{W}_{i}=0$. Note that (134) is reminiscent of the dual coordinate introduced for closed string by us in the case of closed closed string under a similar scenario [22]. We can go further and scenario [22]. We can go further and opt for a first order formalism and consider the Lagrangian density

$$
\widetilde{\mathcal{L}}=\frac{1}{2} \bar{\Sigma}^{i}\left(G_{i j}(\phi)-\gamma_{5} B_{i j}(\phi)\right) \Sigma^{j}-\bar{\Sigma}^{i} D \widetilde{W}_{i}
$$

The $\bar{\Sigma}^{i}$ variation leads to

$$
\left(G_{i j}(\phi)-\gamma_{5} B_{i j}(\phi)\right) \Sigma^{j}=D \widetilde{W}_{i}
$$

and $\widetilde{W}_{i}$ variation implies $\bar{D} \bar{\Sigma}^{i}=0$. Therefore, when $\Sigma^{i}=D W^{i}$ we recover (133). We are in a position to introduce a dual Lagrangian density in terms of the dual superfields, $\widetilde{W}_{i}$ and a set of dual backgrounds $\mathcal{G}^{i j}(\phi)$ and $\mathcal{B}^{i j}(\phi)$; whereas the former of the two backgrounds is symmetric in its indices the latter is antisymmetric.

$$
\mathcal{L}_{\widetilde{W}}=-\frac{1}{2} \bar{D} \widetilde{W}_{i}\left(\mathcal{G}^{i j}(\phi)-\gamma_{5} \mathcal{B}^{i j}(\phi)\right) D \widetilde{W}_{j}
$$


where the two dual backgrounds, $(\mathcal{G}, \mathcal{B})$, are related to the original background fields, $(G, B)$ through the following equations

$$
\mathcal{G}=\left(G-B G^{-1} B\right)^{-1} \text { and } \mathcal{B}=-\left(G-B G^{-1} B\right)^{-1} B G^{-1}
$$

Notice that $\left(G-B G^{-1} B\right)^{-1}$ is symmetric since $\left(G-B G^{-1} B\right)$ is symmetric and it is easy to check that $\mathcal{B}$ is antisymmetric. The equations of motion associated with (137) is

$$
\bar{D}\left(\left[\mathcal{G}(\phi)-\gamma_{5} \mathcal{B}(\phi)\right] D \widetilde{W}\right)=0
$$

Our next step is to write down a pair of equations relating the superfields and their duals which will lead us to T-duality covariant equations of motion. This is facilitated by inspecting the two sets of equations of motion (133) and (139) resulting from the original Lagrangian density and its dual which correspond to two conservation laws. After straight forward and a little tedious calculations [82] we arrive at following two equations

$$
\begin{gathered}
D W^{i}=\gamma_{5}\left(G^{-1} B\right)_{j}^{i} D W^{j}+G^{-1^{i j}} D \widetilde{W}_{j} \\
D \widetilde{W}_{i}=\gamma_{5}\left(\mathcal{G}^{-1} \mathcal{B}\right)_{i}^{j} D \widetilde{W}_{j}+\mathcal{G}_{i j}^{-1} D W^{j}
\end{gathered}
$$

Using (138) in (141) we get two sets of equations relating $D W^{i}$ and $D \tilde{W}_{i}$. Let us define a 2 d-dimensional $O(d, d)$ vector (each one is a superfield) such that

$$
\mathbf{U}=\left(\begin{array}{c}
W^{i} \\
\widetilde{W}_{i}
\end{array}\right)
$$

and a matrix

$$
\mathcal{M}=\left(\begin{array}{cc}
1 G^{-1} & \gamma_{5} G^{-1} B \\
-\gamma_{5} B G^{-1} & 1 G-1 B G^{-1} B
\end{array}\right)
$$

where 1 is the $2 \times 2$ unit matrix and $\gamma_{5}$ is two dimensional diagonal matrix defined earlier. The $\mathcal{M}$ matrix has properties of the familiar $M$-matrix introduced in dimensional reduction of closed bosonic string: $\mathcal{M} \in O(d, d)$ and corresponding metric is $\eta$. The dimensions are further doubled due to the presence of two component Majorana fermions.and is reflected by the appearance of $\mathbf{1}$ and $\gamma_{5}$ in the $\mathcal{M}$-matrix. The two equations (140) and (141) can be combined to a single matrix equation

$$
D \mathbf{U}=\mathcal{M} \eta \mathbf{U}
$$

It follows from the definition of the $O(d, d)$ vector $\mathbf{U}$ that $\bar{D} D \mathbf{U}=0$. It holds by virtue of the fact that the two components of $\mathbf{U}$ satisfy $\bar{D} D W^{i}=0$ and $\bar{D} D \widetilde{W}_{i}=0$ 
from our original equations (they are dual superfields of each other). Therefore, we arrive at an $O(d, d)$ covariant equations of motion for coordinates along compact directions

$$
\bar{D}(\mathcal{M} \eta \mathbf{U})=0
$$

Thus (145) generalizes the closed string $O(d, d)$ covariant equations of motion to NSR superstring [82].

Let us return to the evolution equation for the superfields corresponding to noncompact coordinates

$$
\bar{D}\left[\left(g_{\mu \nu}(\phi)-\gamma_{5} B_{\mu \nu}(\phi)\right) D \phi^{\nu}\right]=0
$$

Notice that due to the dependence of backgrounds on the superfield $\phi$ these are "dynamical" equations unlike the case of compact coordinates which were identified as conservation laws. More important point to note is that these equations are Tduality invariant since the background tensors and these superfields are inert under the action of the noncompact T-duality symmetry group. Therefore, we conclude that the resulting equations of motion for a superstring compactified on $T^{d}$ can be cast in an $O(d, d)$ covariant form. In the next section, we shall consider an illustrative example.

\subsection{Type IIB Compactification on $\mathrm{AdS}_{3} \otimes \mathrm{S}^{3} \otimes \mathrm{T}^{4}$}

We envisage a scenario where our results can be concretely realized [82]. In the presence of NS-NS 3-form flux we can write down a worldsheet action for the case at hand. Notice that $A d S_{3}$ and $S^{3}$ correspond to target space of constant negative and positive curvatures respectively. Therefore, if we introduce appropriate NS-NS three form fluxes, we can describe the Lagrangian in these two sectors as sum of two WZW Lagrangians. The presence of WZ term renders the theory conformally invariant and has the interpretation of the background antisymmetric tensor fields. Moreover, for the present scenario the the associated field strengths are constant. The radii of these two spaces are to be such that the cosmological constants arising from constant positive and negative curvatures of $S^{3}$ and $A d S_{3}$ correspondingly sum up to zero. The worldsheet description of NSR string on $A d S_{3} \otimes S^{3}$ can be formulated as WZW model on group manifolds $S L(2, R) \otimes S U(2)$ as is well known [89, 90, 91, 92, 93, 94]. Thus the full worldsheet action is decomposed into sum of three parts: one corresponds to superconformal theory on $S L(2, R)$ the other being $S U(2)$ and the third part is the one describing a supersymmetric $\sigma$-model on $T^{4}$ as we have discussed in the previous section.

Let us briefly consider bosonic WZW model for $S U(2)$ group whose action is

$$
\begin{aligned}
S_{B} & =\frac{1}{4 \lambda^{2}} \int d \sigma d \tau \operatorname{Tr}\left(\partial_{a} g^{-1}(\sigma, \tau) \partial^{a} g(\sigma, \tau)\right) \\
& +\frac{k}{16 \pi} \int_{B} \operatorname{Tr}\left(g^{-1}(\sigma, \tau) d g(\sigma, \tau) \wedge g^{-1}(\sigma, \tau) d g(\sigma, \tau) \wedge g^{-1}(\sigma, \tau) d g(\sigma . \tau)\right)(1
\end{aligned}
$$


where $g \in S U(2)$ and satisfies the constraint $g g^{\dagger}=\mathbf{1}, \mathbf{1}$ being the unit matrix. Note the following features: (i) $\lambda$ and $k$ are dimensionless coupling constants. For a compact group like $S U(2), k$, the coupling constant appearing in front of the WZ term is quantized for the consistency of the quantized theory. (ii) g should be smoothly extended to a 3-dimensional manifold and its boundary, B, is the worldsheet (actually one should define complex variables in terms of $(\sigma, \tau)$ and this action in those variables in the standard manner.) The theory is conformally invariant at the special point $\lambda^{2}=\frac{4 \pi}{k}$.

The case of (bosonic) string on noncompact $S L(2, R)$ manifold is similar to $S U(2)$ with some differences. (i) The matrix $\tilde{g} \in S L(2, R)$ satisfies the constraint $\tilde{g} \zeta \tilde{g}^{T}=\zeta$ to be contrasted with $g \in S U(2)$ group element. Here $\zeta$ is the $S L(2, R)$ metric with property $\zeta^{2}=-\mathbf{1}$ and it can be chosen to be

$$
\zeta=\left(\begin{array}{cc}
0 & 1 \\
-1 & 0
\end{array}\right)
$$

(ii) In this case the coefficient of WZ, $k$ term need not be quantized.

We shall consider the supersymmetric WZW model for $S U(2)$ from now on. The action is $[89,90,91,92,93,94]$

$$
S=\frac{1}{4 \lambda^{2}} \int d \sigma d \tau d^{2} \theta \bar{D} \mathbf{G}^{\dagger} D \mathbf{G}+\frac{k}{16 \pi} \int d \sigma d \tau d^{2} \theta \int_{0}^{1} d t \mathbf{G}^{\dagger} \frac{\mathbf{d} \mathbf{G}}{d t} \bar{D} \mathbf{G}^{\dagger} \gamma_{5} D \mathbf{G}
$$

The matrix $\mathbf{G}$ defined in the superspace satisfies constraints $\mathbf{G G}^{\dagger}=\mathbf{1}$. In order to define the WZ term as an integral over a three dimensional space one defines extension of the superfield to 3 -dimensions so that $t=0$ corresponds to the boundary i.e. at that point the two dimensional superfield is defined on the worldsheet and two dimensional $\gamma_{5}$ is defined already. Several remarks are in order at this point: (i) We express $\mathbf{G} \in S U(2)$, the matrix in terms of component fields. When the auxiliary field is eliminated from the action and the $d \theta$ integration is done, the resulting action contains quartic fermionic coupling and the theory is not necessarily conformally invariant for arbitrary $\lambda^{2}$ and $k$. (ii) At the special point $\lambda^{2}=\frac{4 \pi}{k}$, theory is conformally invariant and the quartic fermionic coupling disappears. Therefore, for a superstring on a group manifold the two coupling constants are related (and $k$ is quantized). Moreover, at the conformal point, the equations of motion of the superfields (G-matrices), decompose into holomorphic and antiholomorphic parts and the equations of motion take the form of two separate current conservation equations. This feature is most elegantly displayed if we expand the super-matrix in $\mathbf{G}$ in light cone variables as

$$
\mathbf{G}(\sigma, \tau, \theta)=g(\sigma, \tau)\left(\mathbf{1}+i \theta^{+} \psi_{+}(\sigma, \tau)+i \theta^{-} \psi_{-}(\sigma, \tau)+i \theta^{+} \theta^{-} F(\sigma, \theta)\right)
$$

Corresponding light cone superderivatives are

$$
D_{ \pm}=\frac{\partial}{\partial \theta^{ \pm}}-i \theta^{ \pm} \partial_{ \pm} \text {with } \partial_{ \pm}=\partial_{\tau} \pm \partial_{\sigma}
$$


Note that the chiral fermions $\psi_{ \pm}$are matrices taking value in the Lie algebra and $F$ is the auxiliary field. The constraint $G G^{\dagger}=\mathbf{1}$ results in relations between component fields, $g, \psi_{ \pm}$and $F$. At the conformal point the equation of motions become

$$
D_{\mp} \mathbf{J}_{ \pm}=0, \quad \mathbf{J}_{ \pm}=-i \mathbf{G}^{-1} D_{ \pm} \mathbf{G}
$$

We therefore, note that classically we solve for NSR string on $S^{3}$.

The case of NSR string on $A d S_{3}$, proceeds similarly once we take into account the subtleties associated with the noncompact $S L(2, R)$ group.

We have discussed in detail how to construct worldsheet action for compact coordinates in the case of NSR string on $T^{d}$. We showed that the equations of motion can be cast in $O(d, d)$ covariant form since the equations of motion are conservation laws in the superspace.

We argue that the string coordinates and backgrounds, parametrizing target space $A d S_{3}$ and $S^{3}$, transform trivially under the T-duality group associated with compact directions. Therefore, those equations of motion are $O(d, d)$ invariant. This completes our study of T-duality symmetry for type IIB string on $A d S_{3} \otimes S^{3} \otimes T^{4}$.

\subsection{Vertex Operators for Excited States and T-duality}

We study construction of duality symmetric vertex operators of NSR string in this subsection. We have shown, in the preceding section, that for bosonic closed string compactified on $T^{d}$ the vertex operators associated with excited massive states can be expressed in an $O(d, d)$ invariant form. This was achieved in a simple frame work. We worked in the weak field approximation when strings is considered in the background of massive excited states. These vertex operators were first expressed in terms $\sigma$-derivatives of $X^{\mu}$ and the canonical conjugate momenta of the compact coordinates and $\sigma$ and/or $\tau$ derivatives. The vertex operators are required to fulfill following conditions. (i) All vertex operators are required to be $(1,1)$ operators with respect to the stress energy momentum tensors, $\left(T_{++}, T_{--}\right)$, of the free string $[65,66]$. This is a powerful constraint and it leads to the 'equations of motion' and 'gauge conditions' for the massive backgrounds when they are arbitrary functions of string coordinates. (ii) At each mass level one constructs 'vertex functions' from the basic building blocks such as $\partial X^{\hat{\mu}}, \bar{\partial} X^{\hat{\mu}}$ and $\partial$ or $\bar{\partial}$ acting on these building blocks. Note that we do not admits terms like $\partial \bar{\partial} X^{\hat{\mu}}$ or $\bar{\partial} \partial X^{\hat{\mu}}$ in vertex functions since these objects and derivative of such objects vanish as a virtue of free string equations of motion. (iii) The structure of vertex function of a given type, at each mass level, is constrained by the level matching conditions since there is no preferred point on a closed string loop.

(iv) The vertex operator of a given mass level is sum of all such vertex functions. The vertex operator is required to be $(1,1)$; consequently, at a given mass level, the vertex functions are related to each other (see [23] for details). At each mass level there are excitations of various angular momenta of same mass. In other words the states belong to the irreducible representations of $S O(\hat{D}-1)$. (v) When we compactify the 
theory to lower dimensions: $M_{D} \otimes T^{d}$, all the states of a given level are classified according to irreducible representations of $S O(D-1)$ including the states coming from excitations in compact directions (these are all scalars) since total degrees of freedom (at each level) remains the same in both the cases.

We mention in passing that the constraints of conformal invariance need not be imposed at this stage while we are investigating duality symmetries. Those requirements further restrict the structures of of vertex operators and provide useful relations among vertex functions besides imposing mass shell conditions for a given mass level. We intend to derive analogous results for the vertex functions of the excited massive states of NSR string. Notice that for the first excited level on the leading Regge trajectory for NSR string will have a lot more terms compared to (??) since we can construct additional terms which contract with chiral worldsheet fermions. For example, we can have generic terms like

$$
G_{\hat{\mu} \hat{\nu} \hat{\rho} \hat{\lambda} \hat{\delta}}^{(1)} \partial X^{\hat{\mu}} \partial X^{\hat{\nu}} \psi_{-}^{\rho} \psi_{-}^{\hat{\lambda}} \bar{\partial} X^{\hat{\delta}}, G_{\hat{\mu} \hat{\nu} \hat{\rho} \hat{\lambda} \hat{\delta} \hat{\epsilon}}^{(2)} \partial X^{\hat{\mu}} \partial X^{\hat{\nu}} \psi_{-}^{\rho} \psi_{-}^{\hat{\lambda}} \psi_{-}^{\delta} \psi_{-}^{\hat{\epsilon}}
$$

and several other terms where $\partial$ is replaced by $\bar{\partial}$ and $\psi_{-}$is replaced by $\psi_{+}$so long as we ensure, to start with, we have maintained same dimensionality for product of left movers and right movers with respect to the two stress energy momentum tensors. The discrete $Z_{2}$ symmetry alluded to in (122) will be maintained if we take into account all required terms for the vertex operator under considerations. It is quite obvious even keeping track of all terms for the vertex operators of some of the low lying excited massive levels is going to be not very efficient if we want to check the conjectured T-duality for superstrings in terms of the bosonic coordinates and NSR fermions. So far there was no construction of manifestly $O(d, d)$ invariant vertex operators for NSR string along compact directions even for the massless sector i.e. massless scalars that arise from compactification of $G_{\hat{\mu} \hat{\nu}}$ and $B_{\hat{\mu} \hat{\nu}}$.

Therefore, we resort to the superfield approach and consider vertex functions for massive excited states constructed out of the superderivatives of superfields [82]. There are two types of generic vertex functions

(i) $\bar{D} W^{i_{1}} \bar{D} W^{i_{2}} \ldots \bar{D} W^{i_{m}} D W^{j_{1}} D W^{j_{2}} \ldots D W^{j_{m}}$. These correspond to leading Regge trajectories.

(ii) $\bar{D}^{p} W^{i_{1}} \bar{D}^{q} W^{i_{2}} \ldots . \bar{D}^{r} W^{i_{m}} D^{p^{\prime}} W^{j_{1}} D^{q^{\prime}} W^{j_{2}} \ldots D^{r^{\prime}} W^{j_{m}}$ and we require $p+q+r=p^{\prime}+$ $q^{\prime}+r^{\prime}$.

We proceed with following step for the case first case i.e. vertex functions corresponding to leading Regge trajectories which are obtained after the compactification. (I) Recall that $\mathbf{U}$ is an $O(d, d)$ vector, whose upper component is $W^{i}$ and the lower component is its dual $\widetilde{W}_{i}$. Introduce two projection operators [82]

$$
\widetilde{P}_{+}=\left(\begin{array}{ll}
1 & 0 \\
0 & 0
\end{array}\right) \quad \widetilde{P}_{-}=\left(\begin{array}{ll}
0 & 0 \\
0 & 1
\end{array}\right)
$$

Note that $\widetilde{P}_{+} \mathbf{U}=W$. 
(II) Introduce a doublet through the pair $(D, \bar{D})$.

$$
\mathcal{D}=\left(\frac{D}{D}\right)
$$

Then projection operators

$$
\widetilde{\Delta}_{+}=\left(\begin{array}{ll}
1 & 0 \\
0 & 0
\end{array}\right) \quad \widetilde{\Delta}_{-}=\left(\begin{array}{ll}
0 & 0 \\
0 & 1
\end{array}\right)
$$

Note that $\widetilde{P}_{ \pm}$are $2 d \times 2 d$ dimensional projectors whereas $\widetilde{\Delta}_{ \pm}$are $2 \times 2$ projectors. The vertex functions which assume the form given in (i) above can be cast as products of $O(d, d)$ vectors $[82]$

$$
\widetilde{\Delta}_{-} \mathcal{D} \widetilde{P}_{+} \mathbf{U}^{\alpha_{1}} \ldots . \widetilde{\Delta}_{-} \mathcal{D} \widetilde{P}_{+} \mathbf{U}^{\alpha_{m}} \widetilde{\Delta}_{+} \mathcal{D} \widetilde{P}_{+} \mathbf{U}^{\beta_{1}} \ldots . \widetilde{\Delta}_{+} \mathcal{D} \widetilde{P}_{+} \mathbf{U}^{\beta_{m}}
$$

Thus we have an $O(d, d)$ tensor of rank $2 m$. We contract it with a tensor, which depends on spacetime superfield $\phi^{\mu}$ to construct an $O(d, d)$ invariant vertex function for the $n^{t h}$ massive level.

$$
V_{n+1}=T_{\alpha_{1} . . \alpha_{m} \beta_{1} . . \beta_{m}} \widetilde{\Delta}_{-} \mathcal{D} \widetilde{P}_{+} \mathbf{U}^{\alpha_{1}} \ldots . \widetilde{\Delta}_{-} \mathcal{D} \widetilde{P}_{+} \mathbf{U}^{\alpha_{m}} \widetilde{\Delta}_{+} \mathcal{D} \widetilde{P}_{+} \mathbf{U}^{\beta_{1}} \ldots . \widetilde{\Delta}_{+} \mathcal{D} \widetilde{P}_{+} \mathbf{U}^{\beta_{m}}
$$

If the $O(d, d)$ vector transforms as: $U^{\alpha_{1}} \rightarrow \Omega_{\alpha_{1}^{\prime}}^{\alpha_{1}} U^{\alpha_{1}^{\prime}}$, then we require

$$
T_{\alpha_{1}, \ldots \alpha_{m} \beta_{1} . . \beta_{m}} \rightarrow \Omega_{\alpha_{1}}^{\alpha_{1}^{\prime} . .} \Omega_{\alpha_{m}}^{\alpha_{m}^{\prime}} \Omega_{\beta_{1}}^{\beta_{1}^{\prime}} \ldots \Omega_{\beta_{m}}^{\beta_{m}^{\prime}} T_{\alpha_{1}^{\prime} . . \alpha_{m}^{\prime} \beta_{1}^{\prime} \ldots \beta_{m}^{\prime}}
$$

so that the vertex function $V_{n+1}$ is T-duality invariant.

Now we focus attention on the second type of vertex function mentioned in (ii) above. Note that a typical term appearing in the product is like $(\bar{D})^{p}$ and $(D)^{p^{\prime}}$. We can use the projection operators introduced in (I) and (II) above to express products of such terms as $[82]$

$$
\widetilde{\Delta}_{-}^{p} \widetilde{P}_{+} U^{\alpha_{1}} \widetilde{\Delta}_{-}^{q} \widetilde{P}_{+} U^{\alpha_{2}} . . \widetilde{\Delta}_{-}^{r} \widetilde{P}_{+} U^{\alpha_{m}} \widetilde{\Delta}_{+}^{p^{\prime}} \widetilde{P}_{+} U^{\beta_{1}} \widetilde{\Delta}_{+}^{p^{\prime}} \widetilde{P}_{+} U^{\beta_{2}} . . \widetilde{\Delta}_{+}^{p^{\prime}} \widetilde{P}_{+} U^{\beta_{m}}
$$

Now this is an $O(d, d)$ tensor of rank $p+q+r$ satisfying the level matching condition. As in the previous case, we have to just contract with a tensor (which depends on superfield $\phi)$ to get an $O(d, d)$ invariant vertex function.

So far we have left out two other possibilities which we dwell upon now. There are two more types of vertex functions in a given level:

(a) We can have a situation that the vertex function has product of mixed set of operators i.e. some of the superfields correspond to spacetime coordinates and some to compact ones.

$$
T_{\mu_{1} . . \mu_{k} \alpha_{1} . . \alpha_{l} \mu_{1}^{\prime} . . \mu_{k}^{\prime} \alpha_{1}^{\prime} \alpha_{l}^{\prime}}(\phi) \bar{D}^{p} \phi^{\mu_{1}} . . \bar{D}^{q} \phi^{\mu_{k}} \bar{D}^{r} W^{\alpha_{1}} \bar{D}^{s} W^{\alpha_{l}} D^{p^{\prime}} \phi^{\mu_{1}^{\prime}} D^{q^{\prime}} \phi^{\mu_{k}^{\prime}} D^{r^{\prime}} W^{\alpha_{1}^{\prime}} D^{s^{\prime}} W^{\alpha_{l}^{\prime}}(161)
$$


First notice that $\phi^{\mu}$ is inert under T-duality transformations. Similarly, all the spacetime indices of the tensor $T_{\mu \nu \ldots \mu^{\prime} \nu^{\prime} . .}(\phi)$ do not get transformed under T-duality. Moreover, all the spacetime indices of $T$ are contracted with spacetime superfields so that effectively we deal with a tensor with "internal" indices which are contracted with product of building blocks consisting of superderivatives of $W$ 's i.e $\bar{D}$ or $D$ acting on $W^{\prime}$ 's . We already presented a prescription of constructing $O(d, d)$ invariant vertex functions out of such products. Therefore, any arbitrary vertex function of a given massive level can be expressed in an $O(d, d)$ invariant form.

(b) There is another class of vertex functions which are product of the superderivatives of the spacetime superfields only. However, this class of vertex functions are automatically T-duality invariant since the superderivatives $\phi^{\mu}$ and corresponding $\phi$ dependent tensors are not sensitive to $O(d, d)$ transformations.

In conclusion for an NSR string compactified on $T^{d}$, we can express all vertex functions at each massive level in T-duality invariant form.

Another important point deserves attention. Let us consider the the two generic vertex functions represented by (158) and (161). A vertex operator at a given mass level is linear combination of all possible vertex functions consistent with the mass for that level and satisfying the level matching condition. When we demand that, thus constructed vertex operator satisfies $(1,1)$ condition with respect to the stress energy momentum tensor, all the vertex functions are not necessarily independent. The vertex functions satisfy 'equations of motion' which are onshell conditions and satisfy 'transversality' conditions. For a given mass level the tensors given in (161), $T_{\mu_{1}, \mu_{2} . . \alpha_{1} . . \alpha_{l}, \mu_{1}^{\prime}, \mu_{2}^{\prime} . . \alpha_{1}^{\prime} . . \alpha_{l}^{\prime}}$, for example, depends on the superfield, $\phi^{\mu}$, which are along the noncompact directions. These tensors satisfy 'equations of motion' and the transverality condition when we demand that the vertex operators satisfy requirements of superconformal symmetry. Note that of the set of tensors appearing in the construction of vertex functions not all are independent. For detail discussions, in the context of excited levels of closed bosonic string we refer the reader to [23] where T-duality properties were discussed and the equations of motion and transversality conditions were presented. If we were to envisage above issues from the perspective of BRST formalism, the vertex operators will be required to be BRST invariant. As is well known, both the equations of motion and transversality conditions of the vertex operators will be derived as a consequence. We mention in passing that this investigation is focused on NS-NS sector of the theory. It will be interesting to study the RR sector in this approach. This will be taken up in a separate paper.

We address another point in the context of type IIB theory. It is well known that this theory in endowed with S-duality symmetry. Its massless spectrum in NS-NS sector for critical dimension $(\hat{D}=10)$ consists of graviton, $\hat{g}_{\hat{\mu} \hat{\nu}}, 2$-form antisymmetric tensor, $\hat{B}_{\hat{\mu} \hat{\nu}}^{(1)}$ and dilaton, $\hat{\phi}$. The R-R sector is axion, $\hat{\chi}, 2$-form antisymmetric tensor, $\hat{B}_{\hat{\mu} \hat{\nu}}^{(2)}$ and a four form tensor $\hat{C}_{\hat{\mu} \hat{\nu} \hat{\rho} \hat{\lambda}}^{(4)}$ whose field strength is required to be self dual. The effective action for the type IIB theory may be expressed in an S-duality invariant form. When we toroidally compactify the theory to lower dimension, the reduced 
effective can also written in S-duality invariant form. Therefore, starting from NS-NS backgrounds, we can generate RR backgrounds of the reduced theory; however, the reduced tensors of four form $C^{(4)}$ cannot be generated from the NS-NS backgrounds. Let us closely examine the case when type IIB theory is compactified on $T^{4}$ to a six dimensional theory and focus our attention on the moduli and the vector fields coming from reduction of backgrounds ${ }^{2}$. We expect that the massless states coming from NS-NS sector will be classified according to representations of $O(4,4)$. In fact the moduli parametrizes the coset $\frac{O(4,4)}{O(4) \otimes O(4)}$ as was demonstrated by us [22]. The counting is quite simple: the moduli coming from compactification of the graviton and the 2-form antisymmetric tensor add up to 16 as expected. The gauge fields originating from metric and antisymmetric tensor (from NS-NS sector) belong to the vector representation of $O(4,4)$. Indeed, in the NS-NS sector the worldsheet action exhibits presence of all these massless fields, if we follow prescriptions of ref [22]. Let us turn to the R-R sector. There are 9 scalar appearing due to compactification of the 2-form, $B^{(2)}$, the 4 -form $C^{(4)}$ besides the axion. The number of vector fields are eight: four from $B^{(2)}$ and four from $C^{(4)}$. We should also take into account the underlying Sduality symmetry: $S L(2, Z)$. It is more appropriate to classify massless states of the toroidally compactified six dimensional theory combining the states from NS-NS and $\mathrm{RR}$ sector. The arguments are along the same line as classification of branes (hence classifying the background tensors) in the context of toroidal compactification of type IIB theory and M-theory $[96,97,98]$. They belong to representations of $O(5,5)$ from this perspective. Whereas the $25(=16+9)$ moduli parametrize the coset $\frac{O(5,5)}{O(5) \otimes O(5)}$, the $16(=8+8)$ massless vectors belong to the spinor representation of $O(5,5)$. The other backgrounds, in the six dimensional theory, also belong to appropriate representation of this group.

We note that one can study the T-duality attributes of the NS-NS massless backgrounds of the theory compactified on $T^{4}$ in the worldsheet approach presented here. The massive excited backgrounds along the compact direction, in the NS-NS sector, can be coupled to corresponding worldsheet supercoordinates. We are able to express the vertex operators for each of such levels in a manifestly T-duality invariant form. However, it is not possible to construct similar vertex operators for the RR sector in the present formulation.

\section{$5 \quad$ Summary and Conclusions}

We have studied T-duality for compactified closed strings from the worldsheet point of view. It is argued that this approach reveals some of the salient features of the symmetry. We have presented explicit examples to demonstrate some of the interesting properties of this symmetry. Moreover, we reviewed the role the $\mathrm{K}-\mathrm{K}$ modes and winding modes when the string is compactified on $T^{d}$ in the presence of con-

\footnotetext{
${ }^{2}$ I am thankful to John Schwarz for elucidating the arguments presented here
} 
stant $G_{\alpha \beta}$ and $B_{\alpha \beta}$. We demonstrated that the spectrum remains invariant under $O(d, d)$ transformations and showed how the discrete $O(d, d, Z)$ symmetry appears due to periodicity of the compactified closed string coordinates on $T^{d}$. Next we reviewed the duality symmetry of string evolution equations on the worldsheet. We considered the scenario where, the string coordinate $X^{\hat{\mu}}$ are decomposed to sum of noncompact spacetime coordinates, $X^{\mu}, \mu=0,1 \ldots D-1$ and compact coordinates $Y^{\alpha}, \alpha=D, D+1, \ldots \hat{D}-1$ so that $D+d=\hat{D}$. Moreover, all backgrounds are independent of $Y^{\alpha}$ and depend only on $X^{\mu}$. We showed that the equations of motion associated with are $O(d, d)$ covariant and those associated with $X^{\mu}$ are $O(d, d)$ invariant. This sets up a background to provide a better understanding of our approach for the study of duality symmetry associated with excited massive levels.

We have proposed a systematic procedure to obtain T-duality invariant vertex functions for massive levels of a closed bosonic string when it is compactified on $T^{d}$, the $d$-dimensional torus. It is assumed that the tensor fields associated with these vertex operators depend only on the spacetime coordinates, $X^{\mu}(\sigma, \tau)$ and are independent of the compact coordinates, $Y^{\alpha}(\sigma, \tau)$. The duality invariance is manifest for vertex operators of each level once one uses the projection technique to convert $\left\{P, Y^{\prime}\right\}$ to $O(d, d)$ vectors and/or their $\Delta_{ \pm}$derivatives. However, this programme is not complete. We have considered the Hassan-Sen compactification [58] where the metric is decomposed into two diagonal blocks. If we adopt the compactification of [22] then the gauge fields associated with the isometries have to accounted for. Moreover, we considered the target space metric to be flat and have set 2-form tensor to zero in the massless sector. Furthermore, we have ignored the presence of winding modes. A complete study should incorporate all these factors in investigating T-duality properties of excited massive level. If history is a guide, we can conjecture that the vertex operators will continue to be T-duality invariant in the presence all the background field which we have accounted for in the present case. We may recall that simple cases in cosmological scenario were useful in unraveling the $O(d, d)$ symmetry both in the worldsheet approach and in study of (cosmological) effective action. Moreover, Hassan-Sen [58] was an early step to reveal $O(d, d)$ symmetry before more general programme was undertaken [22].

The T-duality symmetry plays an important role in string theory. We expect that these symmetry properties will have important applications. Recall that the T-duality symmetry has been widely applied to obtain new solutions to the background configurations through judicious implementations of the solution generating techniques. Thus given a configuration of massive level background field it will be possible, in principle, to generate another background within the same massive level. Furthermore, there are evidences that massive excited states are endowed with local symmetries. It is worth while to examine the implications of T-duality for those local symmetries. Another point which deserves attention is to study the zero-norm states in this formulation. It is well known that the existence of zero-norm states is quite essential in order that the bosonic string respects Lorentz invariance in critical dimensions i.e. 
$\hat{D}=26$. This issue has been carefully analyzed in $[65,66]$. We expect that these results will continue to hold good when we are dealing with toroidally compactified closed bosonic string.

It is well known that very massive stringy states have possess exponential degeneracy which has played crucial in deriving Bekenstein-Hawking entropy relation for stringy back holes from the counting of microscopic states. This high degree of degeneracy is also instrumental in deducing the thermal nature of emission spectrum of a stringy black hole. We expect that some of supermassive states which also belong to the spectrum of the compactfied string might exhibit symmetry properties which are yet to be discovered.

It is worth while to dwell on another aspect of $O(d, d)$ invariant form of the vertex operator. We recall that a generic vertex function is expressed as product of $O(d, d)$ vectors $\mathcal{W}$ and this product is contracted with a tensor so that the resulting vertex function is $O(d, d)$ invariant. Note, however, that the the product of the $\mathcal{W}$-vectors can be expressed as sum of tensors which are irreducible representations of $O(d, d)$. Thus the vertex function will be sum of terms which are $O(d, d)$ invariant on their own. For sake of definiteness focus on the vertex function associated with the first massive level. This serves as an illustrative example $A_{\alpha \beta, \alpha^{\prime} \beta^{\prime}}^{(1)} \partial Y^{\alpha} \partial Y^{\beta} \bar{\partial} Y^{\alpha^{\prime}} \bar{\partial} Y^{\beta^{\prime}}$. We can always rewrite it as

$$
T_{k l, k^{\prime} l^{\prime}}^{(1)}(X) \mathcal{W}_{+}^{k} \mathcal{W}_{+}^{l} \mathcal{W}_{-}^{k^{\prime}} \mathcal{W}_{-}^{l^{\prime}}
$$

We have distinguished the appearance of the $O(d, d)$ vectors in the expressions for the vertex operators whether they originate from right moving or left moving sector through unprimed and primed indices. The vertex operator $(162)$ is $O(d, d)$ invariant; however, it could be decomposed as sum of contracted tensors belonging to irreducible representations of $O(d, d)$. We first illustrate the point by a simple example from atomic/nuclear physics when one considers familiar multipole operators which usually appear in computations of radiative transitions. The operator $\mathbf{x}^{\mathbf{i}} \mathbf{x}^{\mathbf{j}}$ is decomposed into

$$
\mathbf{x}^{\mathbf{i}} \mathbf{x}^{\mathbf{j}}=\left(\mathbf{x}^{\mathbf{i}} \mathbf{x}^{\mathbf{j}}-\frac{1}{3} \delta^{\mathbf{i j}} \mathbf{x}^{2}\right)+\frac{1}{3} \mathbf{x}^{2} \delta^{\mathbf{i j}}
$$

Note that the first term is the quadrupole operator (traceless). If we construct a scalar $\mathbf{T}_{\mathbf{i j}} \mathbf{x}^{\mathrm{i}} \mathbf{x}^{\mathrm{j}}$; the product decomposes into $\mathbf{T}_{\mathrm{ij}} \mathrm{Q}^{\mathrm{ij}}+\mathbf{T}_{\mathrm{i}}^{\mathrm{i}} \mathbf{x}^{2} ; \mathbf{Q}^{\mathrm{ij}}$ being the quadrupole operator.

Let us examine the tensor structures in (162). $T_{k l, k^{\prime} l^{\prime}}^{(1)}$ is contracted with product of $\mathcal{W}_{+}^{k} \mathcal{W}_{+}^{l}$ and $\mathcal{W}_{-}^{k^{\prime}} \mathcal{W}_{-}^{l^{\prime}}$. Each of these tensors can be decomposed as follows

$$
\mathcal{W}_{+}^{k} \mathcal{W}_{+}^{l}=\left(\mathcal{W}_{+}^{k} \mathcal{W}_{+}^{l}-\frac{1}{2 d} \eta^{k l} \mathcal{W}_{+}^{m} \eta_{m n} \mathcal{W}_{+}^{l}\right)+\frac{1}{2 d} \mathcal{W}_{+}^{m} \eta_{m n} \mathcal{W}_{+}^{l}
$$

and

$$
\mathcal{W}_{-}^{k^{\prime}} \mathcal{W}_{-}^{l^{\prime}}=\left(\mathcal{W}_{-}^{k^{\prime}} \mathcal{W}_{-}^{l^{\prime}}-\frac{1}{2 d} \eta^{k^{\prime} l^{\prime}} \mathcal{W}_{-}^{m^{\prime}} \eta_{m^{\prime} n^{\prime}} \mathcal{W}_{-}^{l^{\prime}}\right)+\mathcal{W}_{-}^{m^{\prime}} \eta_{m^{\prime} n^{\prime}} \mathcal{W}_{-}^{l^{\prime}}
$$


It is obvious that (162) will be composed of sum of terms arising from IRR of $O(d, d)$, taking into account the decompositions (164) and (165), which are generalization of (163) for the case at hand. Therefore, a generic vertex operator, for the $n^{\text {th }}$ massive level, which assumes the form (116), can be converted to an expression of the type (115) using our prescription. Since they are eventually expressed as products of $O(d, d)$ vectors and contracted with suitable tensors. These product of the the $O(d, d)$ vectors will be decomposed into direct sums of the IRR of $O(d, d)$ and thus will contract with the decomposed $O(d, d)$ tensors written as direct sums of IRR tensors. We conclude that all the vertex operators of each massive level will be expressed as sums of IRR's of the T-duality group. Thus as we go to higher and higher levels, we have to deal with higher and higher dimensional representations of this noncompact group. In Section (IV) we focused attention on superstring in the NSR superfield formulation. We presented two results. We considered NSR string in its massless excitations such as graviton and antisymmetric tensor coming from the NS-NS sector. Here we adopted Hassan-Sen compactification and assumed that the backgrounds along compact directions are independent of superfields along compact directions. The equations of motion corresponding to compact superfields can be cast in $O(d, d)$ covariant form once we introduce dual superfields corresponding to compact superfields and define corresponding dual backgrounds. Therefore, we now have derived duality covariant worldsheet equations of motion which is analog of the equations for closed bosonic string.

This step was very useful to construct vertex operators in the NS-NS background for excited massive states. We introduced projection operators to discuss duality properties of vertex functions of massive levels. Therefore, for the Hassan-Sen compactification scheme, we were able to cast the vertex functions in $O(d, d)$ invariant form. However, this programme is not complete so far. We have to address issues in the R-R sector. Next, in order to construct vertex functions for superstrings (type IIA and type IIB), we have to analyze role of GSO projection carefully. An important issue deserves mention here. If we are to carry forward this programme to superstrings, then it is more appropriate to adopt BRST prescription for vertex operators. As is well known, in this formalism, one can a suitable picture. Now the vertex operator is required to be BRST invariant. It is quite likely, when the vertex operators are constructed for superstring, BRST formulation will be economical and more elegant. We presented an example where our proposal for NSR string can be concretely realized. We considered type IIB compactified on $A d S_{3} \otimes S^{3} \otimes T^{4}$. Here $A d S_{3}$ is the spacetime. This simple compactification has certain advantages for us. First of all we can have NS-NS 3-form flux along $S^{3}$. We can have constant NS-NS flux along $A d S_{3}$ with opposite strength. Therefore, in the worldsheet formulation (superfields), we note that the $\sigma$-model associated with $A d S_{3}$ and $S^{3}$ are WZW models on group manifolds $S L(2, R)$ and $S U(2)$ respectively and WZ term accounts for constant $H=d B$ on these manifolds. Moreover, the conformal point the conservation laws in each case are conservation of holomorphic and antiholomorphic super current. Furthermore, 
we can write down the worldsheet action for string along $T^{4}$. Note that the target manifold is a direct product. Therefore, we can write down the vertex operators for excited massive levels following our prescriptions. Although we have focus attention of Ns-NS sector, an S-duality transformation will take us from NS-NS field strengths to the R-R field strength. We mention in passing that all the branes, for this compactification, belong to representation of the $U$-duality group $O(5,5)$. Indeed the coset structure is $\frac{O(5,5)}{O(5) \otimes O(5)}$ However, we have no insight to present worldsheet realization of this symmetry.

There is another interesting approach to dualities in the worldsheet approach. In this formulation the number of string coordinates are doubled and this in scenario some of the nice features of conventional worldsheet approach are not maintained; however, it has been argued that such doubling might have deep significance [56, 99, 100, 101, 102] in string theory. This approach has not made much headway in string theory, the mathematics is indisputable. In this background, recently, a new formulation of field theory has been introduced where $O(D, D)$ invariant action is constructed, $D$ being the number of spacetime dimensions which is doubled [103, 104, 105, 106, 109]. A lot of progress is being made in double field theory with very beautiful mathematical structures. At this stage we have not been able to establish connection of our worldsheet formulation and $O(d, d)$ symmetry with double field theory.

Acknowledgments: I have benefited from discussions with members of String Theory group at Institute of Physics and at NISER; especially from Anirban Basu and Yogesh Srivastava. I would like to thank Caltech and CERN Theoretical Physics groups for providing stimulating atmosphere during various stages of this work and for their very gracious and warm hospitality. I acknowledge very valuable discussions with John Schwarz and Gabriele Veneziano and thank them for sharing their deep insights. I would like to thank the String Community of India for their supports during very trying period. This work is supported by the People of the Republic of India through a Raja Ramanna Fellowship of DAE. 


\section{References}

1. M. B. Green, J. H. Schwarz and E. Witten, Superstring Theory, Vol I and Vol II, Cambridge University Press, 1987.

2. J. Polchinski, String Theory, Vol I and Vol II, Cambridge University Press, 1998.

3. K. Becker, M. Becker and J. H. Schwarz, String Theory and M-Theory: A Modern Introduction, Cambridge University Press, 2007.

4. B. Zwiebach, A First Course in String Theory, Cambridge University Press, 2004.

5. M. Kaku, Introduction to Superstring and M-theory, Springer, 1998.

6. E. Kiritsis, String Theory in Nutshell, Princeton University Press, 2007.

7. M. Gasperini, Elements of String Cosmology, Cambridge University Press, 2007.

8. For reviews: A. Giveon, M. Porrati and E. Rabinovici, Phys. Rep. C244 1994 77.

9. J. H. Schwarz, Lectures on Superstring and M-theory, Nucl. Phys. Suppl. 55B (1997) 1.

10. P. K. Townsend, Four Lectures on M theory, hep-th/9607201.

11. A. Sen, Introduction to Duality Symmetry in String Theory, Les Housches Lecture, 2001.

12. A. Sen, An Introduction to nonperturbative String Theory, hep-th/980205.

13. M. Duff, R. Khuri and J. Lu, Phys. Rep. 259C (1995) 213.

14. J. Maharana, Recent Developments in String Theory, hep-th/9911200.

15. J. E. Lidsey, D. Wands, and E. J. Copeland, Phys. Rep. C337 2000343.

16. M. Gasperini and G. Veneziano, Phys. Rep. C373 20031.

17. K. Igi and S. Matsuda, Phys. Rev. Lett. 18 (1967) 625.

18. R. Dolen, D. Horn and C. Schmid, Phys. Rev. Lett. 19 (1967) 402.

19. V. De Alfaro, S. Fubini, G. Furlan, C. Rossetti, Currents in Hadron Physics, North Holland, 1973. 
20. G. Veneziano, Nuovo Cimento A57 (1968) 190.

21. M. A. Virasoro, Phys. Rev. 177 (1969) 2309.

22. J. Maharana, J. H. Schwarz, Nucl. Phys. B390 (1993) 3.

23. J. Maharana, Nucl. Phys. B843 (2011) 753; arXiv:10101434.

24. J. Scherk and J. H. Schwarz, Nucl. Phys. B194 (1979) 61.

25. K. Kikkawa and M. Yamazaki, Phys. Lett. 149B (1984) 357;

26. N. Sakai and I. Sanda, Prog. Theor. Phys. 75 (1986) 692.

27. V. P. Nair, A Shapere, A. Strominger, and F. Wilczek, Nucl. Phys. 287B (1987) 402.

28. B. Sathiapalan, Phys. Rev. Lett. 58 (1987) 1597.

29. R, Dijkgraaf, E. Verlinde, and H. Verlinde, Commun. Math. Phys. 115 (1988 649.

30. K. S. Narain, Phys. Lett. B169 (1986) 41.

31. K. S. Narain, M. H. Sarmadi, and E. Witten, Nucl. Phys. B279 (1987) 369.

32. P. Ginsparg, Phys. Rev. D35 (1987) 648.

33. P. Gisnparg and C. Vafa, Nucl. Phys. B289 (1987) 414.

34. S. Cecotti, S. Ferrara and L. Giraldello, Nucl. Phys. B308 (1988) 436.

35. R. Brandenberger and C. Vafa, Nucl. Phys. B316 (1988) 391.

36. M. Dine, P.Huet, and N. Seiberg, Nucl. Phys. B322 (1989) 301.

37. J. Molera and B. Ovrut, Phys. Rev. D40 (1989) 1146.

38. G. Veneziano, Phys. Lett. B265 1991287.

39. A. A. Tseytlin and C. Vafa, Nucl. Phys. B372 (1992) 443.

40. M. Rocek and E. Verlinde, Nucl. Phys. 373 (1992) 630.

41. J. H. Horne, G. T. Horowitz, and A. R. Steif, Phys. Rev. Lett. 68 (1992) 568.

42. A.Sen, Phys. Lett. B271 (1992) 295.

43. A. Font, L. Ibanez, D. Lust, and F. Quevedo, Phys. Lett. B249 (1990) 35. 
44. A. Shapere, S. Trivedi, and F. Wilczek, Mod. Phys. Lett. A6 (1991) 2677.

45. S.-J. Rey, Phys. Rev. D43 (1991) 526.

46. J. H. Schwarz and A. Sen, Nucl. Phys. 411 (1994) 35.

47. A. Sen, Int. J. Mod. Phys. A9 (1994) 3707

48. A. Shapere and F. Wilczek, Nucl. Phys. B320 (1989) 669.

49. A. Giveon, E. Rabinovici, and G. Veneziano, Nucl. Phys. B322 (1989) 167.

50. A. Giveon, N. Malkin, and E. Rabinovici, Phys. Lett. B220 (1989) 551.

51. W. Lerche, D. Lüst, and N. P. Warner, Phys. Lett. B231 (1989) 417.

52. K. Meissner and G. Veneziano, Phys. Lett. B267 (1991) 33.

53. K. Meissner and G. Veneziano, Mod. Phys. Lett. A6 (1991) 3397.

54. M. Gasperini, J. Maharana, and G. Veneziano, Phys. Lett. B272 1991277.

55. M. Gasperini, J. Maharana, and G. Veneziano, Phys. Lett. B296 199251.

56. M. J. Duff, Nucl. Phys. B335 (1990) 610.

57. J. Maharana, Phys. Lett. B296 (1992) 65; hep-th/9205015.

58. S. F. Hasan and A. Sen, Nucl. Phys. B375 (1992) 103.

59. S. R. Das and B. Sathiapalan, Phys. Rev. Lett. 57 (1986) 1511.

60. C. Itoi and Y. Watabiki, Phys. Lett. B198 (1987) 486.

61. J. Maharana and G. Veneziano, Nucl. Phys. B283 (1987) 126.

62. J. Maharana and G. Veneziano (unpublished works, 1986, 1991 and 1993).

63. T. Kubota and G. Veneziano, Phys. Lett. B207 (1988) 419

64. J. Maharana, Novel Symmetries of String Theory, in String Theory and Fundamental Interactions, Springer Lecture Notes in Physics, Vol. 737 p525, Ed. G. Gasperini and J. Maharana Springer 2008, Berlin Heidelberg.

65. E. Evans and B. Ovrut, Phys. Rev. D39 (1989) 3016; Phys. Rev. D41 (1990) 3149.

66. J-C. Lee and B. A. Ovrut, Nucl. Phys. B336 (1990) 222.

67. R. Akhoury and Y. Okada; Nucl. Phys. B318 (1989) 176. 
68. B. A. Ovrut and S. Kalyan Rama, Phys. Rev. D45 (1992) 550.

69. E. Accomando, I. Antoniadis, K. Benakli, Nucl. Phys. 579 (2000) 3.

70. M. Bianchi, L. Lopez, R. Richter, JHEP1103 (2011) 051.

71. W-Z. Feng, T. R. Taylor, Nucl. Phys. B856 (2012) 247.

72. W-Z. Feng, D. Lust, O. Schlotterer, S. Stieberger, and T. R. Taylor, Nucl. Phys. B843 (2011) 570.

73. D. J. Gross, P. Mende; Phys. Lett. B197 (1987) 129; Nucl. Phys. B303 (1988) 407.

74. D. Amati, M. Ciafaloni, G. Veneziano, Phys. Lett. B197 (1987) 81.

75. D. Amati, M. Ciafaloni, G. Veneziano, Int. J. Mod. Phys. A3 (1988) 1615.

76. D. Amati, M. Ciafaloni, G. Veneziano, Phys. Lett. B216 (1989) 41.

77. D. Amati, M. Ciafaloni, G. Veneziano,Phys. Lett. B289 (1989) 87.

78. Nucl. Phys. B403 (1993) 707.

79. D. J. Gross, Phys. Rev. Lett. 60 (1988) 1229.

80. A. Sagnotti, Notes on Strings and Higher Spins, arXiv:1112.4285.

81. J. Maharana, Phys. Lett. B695 (2011) 370; arXiv:10101727.

82. J. Maharana, Int. J. Mod. Phys. A27 (2012) 1250140.

83. A. Das and J. Maharana Mod. Phys. Lett. A9 (1994) 1361; hep-th/9401147.

84. W. Siegel, Phys. Rev. D48 (1993) 2826; hep-th/9308138.

85. E. Alvarez,L. Alvarez-Gaume and Y. Lozano, Phys. Lett. B336 (1994); hepth/9406206.

86. S.F. Hassan, Nucl. Phys. B460 (1995) 362; hep-th/9504148.

87. T. Curtright, T. Uematsu and C. Zachos, Nucl. Phys. 469 (1996) 488; hepth/9601096.

88. B. Kulik and R. Roiban, JHEP 0209 (2002) 007; hep-th/0012010.

89. E. Abdalla and M.C.B. Abadalla, Phys. Lett. B152 (1984) 50.

90. E.Abdalla and K. Rothe, Nonperturbative Methods in Two Dimensional Quantum Field Theory, World Scientific, Singapore 1991. 
91. J. Maharana, Mod. Phys. Lett. A20 (2005) 2317.

92. P. di Vecchia, V. G. Knizhnik, J. L. Peterson and P. Rossi, Nucl. Phys. B253 (1985) 701.

93. E. Braaten, T. Curtright and C. Zachos, Nucl. Phys. B260 (1984) 630.

94. For a review see O. Aharony, S. S. Gubser, J. Maldacena, H. Ooguri and Y. Oz Phys. Rep. C323 (2000) 183.

95. J. Maharana, Phys. Lett. B695 (2011) 370; arXiv:10101727.

96. J. H. Schwarz, Phys. Lett. B360 (1995) 13, arXiv:hep-th/9508143.

97. J. H. Schwarz, Phys. Lett. B367 (1996) 97, arXiv:hep-th/9509148.

98. J. Maharana, Phys. Lett. B372 (1996) 53, arXiv:hep-th/9511159.

99. E. Witten, Phys. Rev. Lett. 61 (1988) 670.

100. A. A. Tseytlin, Phys. Lett. B242 (1990) 163.

101. A. A. Tseytlin, Nucl. Phys. B350 (1991) 395.

102. A. A. Tseytlin, Phys. Rev. Lett. 66 (1991) 545.

103. T. Kugo and B. Zwiebach, Prog. Th. Phys. 87 (1992) 801.

104. C. Hull and B. Zwiebach, JHEP, 0909 (2009) 099, arXiv:0904.4664.

105. C. Hull and B. Zwiebach, JHEP 0909 (2009) 090, arXiv:0908.1792.

106. A. Dabholkar and C. Hull, JHEP 0605 (2006)009, arXiv:hep-th/0512005.

107. O. Hohm, C. Hull and B. Zwiebach, JHEP 1008 (2010) 008, arXiv:1006.4823.

108. O. Hohm, S. K. Kwak and B. Zwiebach, Double Field Theory of Type II Strings, arXiv:1107.0008.

109. D. S. Berman and D. C. Thompson, Phys. Lett. B662 (2008) and references therein. 